TRANSACTIONS OF THE

AMERICAN MATHEMATICAL SOCIETY

Volume 357, Number 11, Pages 4607-4632

S 0002-9947(04)03603-7

Article electronically published on December 9, 2004

\title{
NONAUTONOMOUS KATO CLASSES OF MEASURES AND FEYNMAN-KAC PROPAGATORS
}

\author{
ARCHIL GULISASHVILI
}

\begin{abstract}
The behavior of the Feynman-Kac propagator corresponding to a time-dependent measure on $R^{n}$ is studied. We prove the boundedness of the propagator in various function spaces on $R^{n}$, and obtain a uniqueness theorem for an exponentially bounded distributional solution to a nonautonomous heat equation.
\end{abstract}

\section{INTRODUCTION}

In this paper, we develop the $L^{p}$-theory for the Feynman-Kac propagator, corresponding to a nonautonomous heat equation of the following form:

$$
\frac{\partial u}{\partial t}=\frac{1}{2} \Delta u-\mu(t) u \text {. }
$$

In (1), $\mu=\{\mu(t): 0 \leq t \leq T\}$ is a family of distributions on $R^{n}$ such that for every $t \in[0, T]$ and every open ball $B \subset R^{n}$, the restriction of $\mu(t)$ to $B$ is a finite signed Borel measure. We will call such families time-dependent measures. We will also study the behavior of Feynman-Kac propagators in various spaces of continuous functions on $R^{n}$.

A two-parametric family $\{U(t, \tau): 0 \leq \tau \leq t \leq T\}$ of bounded linear operators on the space $L^{p}\left(R^{n}\right)$ with $1 \leq p \leq \infty$ is called an evolution family, provided the following conditions hold:

(1) $U(t, \tau)=U(t, \lambda) U(\lambda, \tau)$ for $0 \leq \tau \leq \lambda \leq t \leq T$.

(2) $U(\tau, \tau)=I$ for $0 \leq \tau \leq T$, where $I$ stands for the identity operator on $L^{p}$.

(3) For every $f \in L^{p}$, the $L^{p}$-valued function $(t, \tau) \rightarrow U(t, \tau) f$ is continuous for $0 \leq \tau \leq t \leq T$. In the case $p=\infty$, we requre the weak* continuity in $L^{\infty}$ instead of the strong continuity.

An evolution family $U$ is called a propagator for equation (1), if in addition to conditions (1)-(3), the following condition holds:

(4) For every $\tau$ such that $0 \leq \tau<T$, the function $u(t)=U(t, \tau) f$ with $t \in[\tau, T]$ is a solution in the $D^{\prime}\left((\tau, T) \times R^{n}\right)$-sense to the initial value problem

$$
\left\{\begin{array}{l}
\frac{\partial u}{\partial t}=\frac{1}{2} \Delta u-\mu(t) u, \\
u(\tau)=f .
\end{array}\right.
$$

One of our objectives in the present paper is to study various classes of timedependent measures (see Section 2). Two of these classes, $\mathcal{P}_{n, T}$ and $\mathcal{P}_{n, T}^{*}$, will be

Received by the editors October 10, 2003 and, in revised form, December 22, 2003.

2000 Mathematics Subject Classification. Primary 35K15; Secondary 60H30.

Key words and phrases. Nonautonomous heat equation, classes of time-dependent measures, Feynman-Kac propagators, time-dependent additive functionals. 
especially important throughout the paper. It will be shown in Section 4 that if $\mu \in \mathcal{P}_{n, T}$ and $1<p \leq \infty$, then the family of linear operators $U_{\mu}$ defined by

$$
U_{\mu}(t, \tau) f(x)=E_{x} f\left(B_{t-\tau}\right) \exp \left\{-C_{\mu}(t-\tau, t)\right\}, \quad 0 \leq \tau \leq t \leq T,
$$

is a propagator for equation (1). In formula (2), $E_{x}$ denotes the expectation in the Wiener space, $B_{t}$ stands for a standard Brownian motion, and $C_{\mu}$ is a timedependent additive functional of the Brownian motion, corresponding to $\mu$ (for the definition of $C_{\mu}$ see Section 3). The functional $C_{\mu}$ satsfies the following condition:

$$
\lim _{k \rightarrow \infty} \sup _{h: 0 \leq h \leq T} \sup _{x \in R^{n}} E_{x} \sup _{t: 0 \leq t \leq h}\left|C_{\mu}(t, h)-\int_{0}^{t} e^{\frac{1}{2 k} \Delta} \mu(h-s)\left(B_{s}\right) d s\right|^{2}=0 .
$$

The family $U_{\mu}$ is called the Feynman-Kac propagator for equation (1).

Together with equation (1), we will study its dual equation,

$$
\frac{\partial w}{\partial \tau}=-\frac{1}{2} \Delta w+\mu(\tau) w .
$$

Next we will define backward propagators for equation (3). Let $Y(t, \tau), 0 \leq \tau \leq$ $t \leq T$, be a family of bounded linear operators on the space $L^{p}$, and let $\mu=\{\mu(t)$ : $0 \leq t \leq T\}$ be a time-dependent measure. Put

$$
\nu(t)=\mu(T-t), 0 \leq t \leq T .
$$

If the family of operators $U$, defined by $U(t, \tau)=Y(T-\tau, T-t)$ for $0 \leq \tau \leq t \leq T$, is an evolution family on $L^{p}$, then $Y$ is called a backward evolution family. If the family $U$ is a propagator for the heat equation $\frac{\partial u}{\partial t}=\frac{1}{2} \Delta u-\nu(t) u$, then the family $Y$ is called a backward propagator for equation (3). It is clear that if $Y$ is a backward propagator for equation (3), then for every $t$ such that $0<t \leq T$, the function $w(\tau)=Y(t, \tau) f$ with $\tau \in[0, t]$ is a solution in the $D^{\prime}\left((0, t) \times R^{n}\right)$-sense to the following terminal value problem:

$$
\left\{\begin{array}{l}
\frac{\partial w}{\partial \tau}=-\frac{1}{2} \Delta w+\mu(\tau) w \\
w(t)=f .
\end{array}\right.
$$

Let $\mu \in \mathcal{P}_{n, T}^{*}$ and $1<p \leq \infty$. Then the family of linear operators $\left\{Y_{\mu}\right\}$ defined by

$$
Y_{\mu}(t, \tau) f(x)=E_{x} f\left(B_{t-\tau}\right) \exp \left\{-A_{\mu}(t-\tau, \tau)\right\}, \quad 0 \leq \tau \leq t \leq T,
$$

is a backward propagator for equation (3) (see Section 4). In (5), $A_{\mu}$ is a timedependent functional of the Brownian motion, corresponding to $\mu$ (for the definition of $A_{\mu}$ see Section 3). The functional $A_{\mu}$ satisfies the following condition:

$$
\lim _{k \rightarrow \infty} \sup _{h: 0 \leq h \leq T} \sup _{x \in R^{n}} E_{x} \sup _{t: 0 \leq t \leq T-h}\left|A_{\mu}(t, h)-\int_{0}^{t} e^{\frac{1}{2 k} \Delta} \mu(s+h)\left(B_{s}\right) d s\right|^{2}=0 .
$$

The family of operators $Y_{\mu}$ given by (5) is called the backward Feynman-Kac propagator for equation (3). It is also possible to define the family $Y_{\mu}$ in the case $\mu \in \mathcal{P}_{n, T}$ and the family $U_{\mu}$ in the case $\mu \in \mathcal{P}_{n, T}^{*}$. These definitions will be given in Remark 10.

Formulas (2) and (5) are based on the Feynman-Kac formula for Schrödinger semigroups. The theory of Schrödinger semigroups associated with Kato class potentials was developed by Aizenman and Simon (see [AS, S] ). More information on Schrödinger semigroups can be found in $\mathrm{DvC}, \mathrm{JL}$. Schrödinger semigroups and 
Feynman-Kac propagators have many similarities. However, there are also significant differences between them, and one of our objectives in this paper is to explain these differences. For instance, instead of the Kato class in the theory of Schrödinger semigroups, two classes of time-dependent measures, $\mathcal{P}_{n, T}$ and $\mathcal{P}_{n, T}^{*}$, arise naturally in the theory of Feynman-Kac propagators. The first of them controls the behavior of the Feynman-Kac propagator, while the second one is related to the backward Feynman-Kac propagator. Another difference between Schrödinger semigroups and Feynman-Kac propagators is the following: Schrödinger semigroups with Kato class potentials are always bounded on $L^{1}$, which is in general false for Feynman-Kac propagators. It will be shown in Section 4 that there is a time-dependent function $V \in \mathcal{P}_{3,1}$ such that $U_{\mu}(t, 0) \notin L\left(L^{1}, L^{1}\right)$ for all $0<t \leq 1$.

Much literature is devoted to perturbations of second order partial differential operators by time-independent or time-dependent potentials and to the corresponding elliptic and parabolic equations (see [AM, BM1, BM2, FLP $[\mathrm{F}, \mathrm{Ge}, \mathrm{H}] \mathrm{LP}, \mathrm{N}$, QZ1, QZ2, RRSV, SV, Z] and the references therein). Interesting results concerning the existence of the fundamental function for a second order parabolic partial differential equation with coefficients from nonautonomous Kato classes were obtained in a recent paper of Liskevich and Semenov LS. The Feynman-Kac propagator for the heat equation $\frac{\partial u}{\partial t}=\frac{1}{2} \Delta u-V(t) u$, where $V$ is a function on $[0, T] \times R^{n}$ belonging to the class $\mathcal{P}_{n, T}$, was studied in [Gu3].

We now give an overview of the results obtained in the present paper. Section 2 is devoted to the study of various classes of time-dependent measures. These classes generalize the Kato class of measures (see [AS, BM1, DvC, F, Gu1, JL, S, V1, V2, Z for more information on the Kato classes of functions and measures), the enlarged Kato class (see [V1, V2, see also Gu1, GK]), and the nonautonomous Kato classes of functions (see Gu3, LS, N, QZ1, QZ2, RRSV, SV]). Our main result in Section 2 is a characterization of classes of time-dependent measures in terms of the corresponding potential operators (see Theorem 1). For the Kato classes of functions and measures, this result was obtained in Gu1, GK].

In Section 3, we introduce and study the additive functionals $C_{\mu}$ and $A_{\mu}$ that were used in formulas (2) and (5). The existence and uniqueness result for these functionals (Theorem 2) is standard. Its proof was influenced by the proof of Theorems 5.11 and 5.1.2 in $[\mathrm{F}]$.

In Section 4, we gather our main results concerning Feynman-Kac propagators. Here we prove the existence theorem for the propagator $U_{\mu}$ with $\mu \in \mathcal{P}_{n, T}$ (see Theorem 3 ) and the $\left(L^{p}-L^{q}\right)$-smoothing theorem for $U_{\mu}$ (see Theorem 4). We also study the behavior of the Feynman-Kac propagator in the spaces of continuous functions on $R^{n}$. We prove that $U_{\mu}$ maps the space $L^{\infty}$ into the space $B C$ of bounded continuous functions (see Theorem 6). This property of $U_{\mu}$ is called the strong Feller property. Moreover, the propagator $U_{\mu}$ is bounded on the space $B U C$ of bounded uniformly continuous functions and also on the space $C_{\infty}$ of continuous functions vanishing at infinity. The boundedness on the space $C_{\infty}$ is called the Feller property of $U_{\mu}$ (see Theorem 7). These facts are well known for Schrödinger semigroups (see $[\mathrm{S}]$ ).

Section 5 of the present paper is devoted to uniqueness problems. Here we prove the uniqueness of an exponentially bounded distributional solution to problem (1) (see Theorem 8). This theorem is a generalization of a result concerning the uniqueness of an exponentially bounded classical solution to the one-dimensional heat 
equation, $\frac{\partial u}{\partial t}=\Delta u$, which was discussed by Titchmarsh in [Ti], p. 282. A stronger result in the classical case is due to Tikhonov [T]. He proved the uniqueness theorem for a classical solution, satisfying the following condition: $|u(t, x)| \leq M \exp \left\{a x^{2}\right\}$ for $(t, x) \in[0, T] \times R^{1}$. We do not know whether our Theorem 8 holds for a distributional solution satisfying Tikhonov's condition in $R^{n}$. In Section 5 , we also prove that the Feynman-Kac propagator is the unique propagator for equation (1), for which the $\left(L^{p}-L^{\infty}\right)$-smoothing condition holds (see Theorem 9). The uniqueness problem for general second order parabolic partial differential equations was studied by numerous authors. In [A], the weak solutions to equations with coefficients from the spaces $L^{q}\left(I ; L^{p}\right)$ were considered. In QZ1, the uniqueness of a uniformly bounded solution to the heat equation with a time-dependent potential $V=\{V(t)\}$ from the parabolic Kato class (this class is similar to our class $\mathcal{P}_{n, T} \cap \mathcal{P}_{n, T}^{*}$ ) was established. In [LS], the uniqueness of a weak solution to a general second order parabolic equation with coefficients from nonautonomous Kato classes was shown. However, additional restrictions were imposed in [LS] on the behavior of the solutions. For instance, in the case of the heat equation with a potential from the parabolic Kato class, the additional condition is as follows: $u V \in L^{1}\left([\tau, T] \times R^{n}\right)$ (see Lemma 4.7 in [LS]). This condition is not necessarily satisfied in the case $V \in \mathcal{P}_{n, T}$.

Finally, we would like to mention that most of the results obtained in this paper were announced in Gu2.

\section{Classes of time-Dependent measures}

We begin this section with the definition of potentials of time-dependent measures. For $\mu=\{\mu(t): 0 \leq t<\infty\}$, we put

$$
\tilde{M}_{\mu}(h, r, x)=\int_{0}^{h} e^{-s} e^{\frac{s}{2} \Delta} \mu(r-s)(x) d s, 0 \leq h<\infty, \quad h \leq r<\infty, x \in R^{n},
$$

and

$$
\tilde{N}_{\mu}(h, r, x)=\int_{0}^{h} e^{-s} e^{\frac{s}{2} \Delta} \mu(s+r)(x) d s, 0 \leq h \leq \infty, 0 \leq t<\infty, \quad x \in R^{n} .
$$

For $\mu=\{\mu(t): 0 \leq t \leq T\}$, we put

$$
\begin{aligned}
& L_{\mu}(h, r, x)=\int_{0}^{h} e^{\frac{s}{2} \Delta} \mu(r-s)(x) d s, 0 \leq h \leq r \leq T, x \in R^{n}, \\
& S_{\mu}(h, r, x)=\int_{0}^{h} e^{\frac{s}{2} \Delta} \mu(s+r)(x) d s, 0 \leq r+h \leq T, \quad x \in R^{n} .
\end{aligned}
$$

We also put

$$
M_{\mu}(t, x)=L_{\mu}(t, t, x), \quad 0 \leq t \leq T, x \in R^{n},
$$

and

$$
N_{\mu}(t, x)=S_{\mu}(T-t, t, x), \quad 0 \leq t \leq T, x \in R^{n} .
$$

In the formulas above, we assume that the integrals make sense. 
The next definitions concern classes of time-dependent measures.

Definition 1. The classes $\hat{\mathcal{P}}_{n, T}$ and $\hat{\mathcal{P}}_{n, T}^{*}$ are defined as follows:

$$
\begin{aligned}
& \hat{\mathcal{P}}_{n, T}=\left\{\mu=\{\mu(t) ; 0 \leq t \leq T\}: \sup _{t: 0 \leq t \leq T} \sup _{x \in R^{n}} M_{|\mu|}(t, x)<\infty\right\}, \\
& \hat{\mathcal{P}}_{n, T}^{*}=\left\{\mu=\{\mu(t) ; 0 \leq t \leq T\}: \sup _{t: 0 \leq t \leq T} \sup _{x \in R^{n}} N_{|\mu|}(t, x)<\infty\right\} .
\end{aligned}
$$

Definition 2. The classes $\hat{\mathcal{P}}_{n, \infty}$ and $\hat{\mathcal{P}}_{n, \infty}^{*}$ are defined as follows:

$$
\begin{aligned}
& \hat{\mathcal{P}}_{n, \infty}=\left\{\mu=\{\mu(t) ; 0 \leq t<\infty\}: \sup _{t: t \geq 0} \sup _{x \in R^{n}} \tilde{M}_{|\mu|}(t, t, x)<\infty\right\}, \\
& \hat{\mathcal{P}}_{n, \infty}^{*}=\left\{\mu=\{\mu(t) ; 0 \leq t<\infty\}: \sup _{t: t \geq 0} \sup _{x \in R^{n}} \tilde{N}_{|\mu|}(\infty, t, x)<\infty\right\} .
\end{aligned}
$$

Definition 3. The following formulas define more classes of time-dependent measures:

$$
\begin{gathered}
\mathcal{P}_{n, \infty}=\hat{\mathcal{P}}_{n, \infty} \cap\left\{\mu: \lim _{t \rightarrow 0+} \sup _{h: h \geq t} \sup _{x \in R^{n}} L_{|\mu|}(t, h, x)=0\right\}, \\
\mathcal{P}_{n, T}=\hat{\mathcal{P}}_{n, T} \cap\left\{\mu: \lim _{t \rightarrow 0+} \sup _{h: t \leq h \leq T} \sup _{x \in R^{n}} L_{|\mu|}(t, h, x)=0\right\}, \\
\mathcal{P}_{n, \infty}^{*}=\hat{\mathcal{P}}_{n, \infty}^{*} \cap\left\{\mu: \lim _{t \rightarrow 0+} \sup _{h: h \geq 0} \sup _{x \in R^{n}} S_{|\mu|}(t, h, x)=0\right\}, \\
\mathcal{P}_{n, T}^{*}=\hat{\mathcal{P}}_{n, T}^{*} \cap\left\{\mu: \lim _{t \rightarrow 0+} \sup _{h: 0 \leq h \leq T-t} \sup _{x \in R^{n}} S_{|\mu|}(t, h, x)=0\right\} .
\end{gathered}
$$

The classes $\mathcal{P}_{n, T}$ and $\mathcal{P}_{n, T}^{*}$ were introduced in [Gu3] in the case of time-dependent functions. In the present paper we use the same symbols for the corresponding classes of time-dependent measures.

Remark 1. Let $\mu=\{\mu(t): 0 \leq t \leq T\}$ be a time-dependent measure, and denote by $\tilde{\mu}$ its extension to $[0, \infty)$ by the zero measure. Then we have $\mu \in \hat{\mathcal{P}}_{n, T} \Longleftrightarrow$ $\tilde{\mu} \in \hat{\mathcal{P}}_{n, \infty}$. Similar equivalences hold for all the classes of time-dependent measures defined above.

Remark 2. If $\mu$ and $\nu$ are related by formula (4), then $\mu \in \hat{\mathcal{P}}_{n, T} \Leftrightarrow \nu \in \hat{\mathcal{P}}_{n, T}^{*}$ and $\mu \in \mathcal{P}_{n, T} \Leftrightarrow \nu \in \mathcal{P}_{n, T}^{*}$

Remark 3. The classes $\mathcal{P}_{n, T}$ and $\mathcal{P}_{n, T}^{*}$ do not coincide (see Gu3]).

The following characterization of the Kato class of measures $\tilde{K}_{n}$ was obtained in Gu3 (see also GK] where the Kato class $K_{n}$ was considered):

$$
\mu \in \tilde{K}_{n} \Longleftrightarrow(I-\Delta)^{-1}|\mu| \in B U C .
$$

The next theorem is a generalization of equivalence (6) to the case of time-dependent measures.

Theorem 1. (i) A time-dependent measure $\mu \in \hat{\mathcal{P}}_{n, \infty}$ belongs to the class $\mathcal{P}_{n, \infty}$ if and only if the function $(h, x) \rightarrow \tilde{M}_{|\mu|}(h, h, x)$ is uniformly continuous on $[0, \infty) \times$ $R^{n}$.

(ii) A time-dependent measure $\mu \in \hat{\mathcal{P}}_{n, \infty}^{*}$ belongs to the class $\mathcal{P}_{n, \infty}^{*}$ if and only if the function $(h, x) \rightarrow \tilde{N}_{|\mu|}(\infty, h, x)$ is uniformly continuous on $[0, \infty) \times R^{n}$. 
(iii) A time-dependent measure $\mu \in \hat{\mathcal{P}}_{n, T}$ belongs to the class $\mathcal{P}_{n, T}$ if and only if the function $M_{|\mu|}$ is uniformly continuous on $[0, T] \times R^{n}$.

(iv) A time-dependent measure $\mu \in \hat{\mathcal{P}}_{n, T}^{*}$ belongs to the class $\mathcal{P}_{n, T}^{*}$ if and only if the function $N_{|\mu|}$ is uniformly continuous on $[0, T] \times R^{n}$.

Proof. We will prove only part (ii) of Theorem 1 . The proof of the remaining parts is similar.

Let $\mu \in \mathcal{P}_{n, \infty}^{*}$. Then we have

$$
\begin{gathered}
\sup _{h \geq 0} \sup _{x \in R^{n}}\left|\tilde{N}_{|\mu|}(\infty, h, x)-e^{-t} e^{\frac{t}{2} \Delta} \tilde{N}_{|\mu|}(\infty, t+h, x)\right| \\
\leq \sup _{h \geq 0} \sup _{x \in R^{n}} \int_{0}^{t} e^{\frac{s}{2} \Delta}|\mu(s+h)|(x) d s \rightarrow 0
\end{gathered}
$$

as $t \rightarrow 0+$. Since

$$
\sup _{x \in R^{n}} \tilde{N}_{|\mu|}(\infty, t+h, x)<\infty
$$

we get

$$
e^{-t} e^{\frac{t}{2} \Delta} \tilde{N}_{|\mu|}(\infty, t+h, \cdot) \in B U C .
$$

It follows from $(7)$ and $(8)$ that $\tilde{N}_{|\mu|}(\infty, h, \cdot) \in B U C$ for all $h \geq 0$.

Let $t, h \geq 0$ and $x, y \in R^{n}$. Then

$$
\begin{aligned}
\tilde{N}_{|\mu|}(\infty, & h, x+y)-\tilde{N}_{|\mu|}(\infty, h, x) \\
= & \tilde{N}_{|\mu|}(\infty, h, x+y)-e^{-t} e^{\frac{t}{2} \Delta} \tilde{N}_{|\mu|}(\infty, t+h, x+y) \\
& +e^{-t} e^{\frac{t}{2} \Delta} \tilde{N}_{|\mu|}(\infty, t+h, x+y)-e^{-t} e^{\frac{t}{2} \Delta} \tilde{N}_{|\mu|}(\infty, t+h, x) \\
& +e^{-t} e^{\frac{t}{2} \Delta} \tilde{N}_{|\mu|}(\infty, t+h, x)-\tilde{N}_{|\mu|}(\infty, h, x) .
\end{aligned}
$$

Using (7), we see that for every $\epsilon>0$ there exists $t_{0}>0$ such that for all $0<t \leq t_{0}$,

$$
\begin{aligned}
& \sup _{h \geq 0} \sup _{x, y \in R^{n}}\left|\tilde{N}_{|\mu|}(\infty, h, x+y)-e^{-t} e^{\frac{t}{2} \Delta} \tilde{N}_{|\mu|}(\infty, t+h, x+y)\right| \\
& \quad+\sup _{h \geq 0} \sup _{x \in R^{n}}\left|\tilde{N}_{|\mu|}(\infty, h, x)-e^{-t} e^{\frac{t}{2} \Delta} \tilde{N}_{|\mu|}(\infty, t+h, x)\right| \leq \frac{\epsilon}{2} .
\end{aligned}
$$

Fix $t>0$ for which (10) holds. Then (9) and (10) imply

$$
\begin{array}{r}
\sup _{h \geq 0} \sup _{x \in R^{n}}\left|\tilde{N}_{|\mu|}(\infty, h, x+y)-\tilde{N}_{|\mu|}(\infty, h, x)\right| \\
\leq \frac{\epsilon}{2}+\sup _{h \geq 0} \sup _{x \in R^{n}}\left|e^{\frac{t}{2} \Delta} \tilde{N}_{|\mu|}(\infty, t+h, x+y)-e^{\frac{t}{2} \Delta} \tilde{N}_{|\mu|}(\infty, t+h, x)\right| .
\end{array}
$$

It follows from the properties of the heat kernel and from the fact that the set $\left\{\tilde{N}_{|\mu|}(\infty, t+h): h \geq 0\right\}$ is bounded in $C\left(R^{n}\right)$ that the last term on the right-hand side of (11) does not exceed $\frac{\epsilon}{2}$ for $|y|<\delta$ where $\delta$ is a small number. Hence,

$$
\sup _{h \geq 0} \sup _{x \in R^{n}}\left|\tilde{N}_{|\mu|}(\infty, h, x+y)-\tilde{N}_{|\mu|}(\infty, h, x)\right| \rightarrow 0
$$

as $y \rightarrow 0$. Next we see that for all $t \geq 0$,

$$
\begin{gathered}
\sup _{h \geq 0} \sup _{x \in R^{n}}\left|\tilde{N}_{|\mu|}(\infty, t+h, x)-\tilde{N}_{|\mu|}(\infty, h, x)\right| \\
\leq \sup _{h \geq 0} \sup _{x \in R^{n}}\left|\tilde{N}_{|\mu|}(\infty, h, x)-e^{-t} e^{\frac{t}{2} \Delta} \tilde{N}_{|\mu|}(\infty, t+h, x)\right| \\
(13)+\sup _{\tau \geq 0} \sup _{x \in R^{n}}\left|e^{-t} e^{\frac{t}{2} \Delta} \tilde{N}_{|\mu|}(\infty, \tau, x)-\tilde{N}_{|\mu|}(\infty, \tau, x)\right|=I_{1}(t)+I_{2}(t) .
\end{gathered}
$$


It follows from (7) that $I_{1}(t) \rightarrow 0$ as $t \rightarrow 0+$. Let $F \in B U C$. Then for every $\epsilon>0$ and $t>0$ we have

$$
\left\|F-e^{\frac{t}{2} \Delta} F\right\|_{\infty} \leq \sup _{x, y \in R^{n}:|x-y| \leq \epsilon}|F(x)-F(y)|+2|| F \|_{\infty} \int_{|z|>\epsilon} G_{t}(z) d z,
$$

where $G_{t}$ denotes the heat kernel on $R^{n}$. Using (12) and (14), we get $I_{2}(t) \rightarrow 0+$ as $t \rightarrow 0+$. Next (13) gives

$$
\sup _{h \geq 0} \sup _{x \in R^{n}}\left|\tilde{N}_{|\mu|}(\infty, t+h, x)-\tilde{N}_{|\mu|}(\infty, h, x)\right| \rightarrow 0
$$

as $t \rightarrow 0+$. Combining (12) and (15), we obtain

$$
\tilde{N}_{|\mu|}(\infty, \cdot, \cdot) \in B U C\left([0, \infty) \times R^{n}\right) .
$$

Now assume that the previous condition holds. Then (12) and (15) also hold. We have

$$
\begin{array}{r}
\sup _{h \geq 0} \sup _{x \in R^{n}}\left|\tilde{N}_{|\mu|}(\infty, h, x)-e^{-t} e^{\frac{t}{2} \Delta} \tilde{N}_{|\mu|}(\infty, t+h, x)\right| \\
\leq \sup _{h \geq 0} \sup _{x \in R^{n}}\left|\tilde{N}_{|\mu|}(\infty, h, x)-\tilde{N}_{|\mu|}(\infty, t+h, x)\right| \\
+\sup _{h \geq 0} \sup _{x \in R^{n}}\left|\tilde{N}_{|\mu|}(\infty, t+h, x)-e^{-t} e^{\frac{t}{2} \Delta} \tilde{N}_{|\mu|}(\infty, t+h, x)\right| \\
\leq \sup _{h \geq 0} \sup _{x \in R^{n}}\left|\tilde{N}_{|\mu|}(\infty, h, x)-\tilde{N}_{|\mu|}(\infty, t+h, x)\right| \\
+\sup _{\tau \geq 0} \sup _{x \in R^{n}}\left|\tilde{N}_{|\mu|}(\infty, \tau, x)-e^{-t} e^{\frac{t}{2} \Delta} \tilde{N}_{|\mu|}(\infty, \tau, x)\right| \\
=J_{1}(t)+J_{2}(t) .
\end{array}
$$

Condition (15) is the same as $\lim _{t \rightarrow 0+} J_{1}(t)=0$. It follows from (12) and (14) that $\lim _{t \rightarrow 0+} J_{2}(t)=0$. Now using $(16)$, we get $\mu \in \mathcal{P}_{n, \infty}^{*}$.

This completes the proof of Theorem 1.

Next we will discuss examples of singular time-dependent measures in the class $\mathcal{P}_{n, T}$. For the sake of simplicity, we will restrict ourselves to the case $n=1$. In this case, the Dirac measure $\delta_{y}$, concentrated at the point $y \in R^{1}$, belongs to the Kato class of measures $\tilde{K}_{1}$. For $n \geq 2$, one may use appropriate singular measures from the class $\tilde{K}_{n}$ (see examples in [Gu1]).

Let $n=1$, and for every $\alpha>0$ define a time-dependent measure by $\mu_{\alpha}=\left\{t^{-\alpha} \delta_{0}\right.$ : $0 \leq t \leq 1\}$. Then we have $\mu_{\alpha} \in \hat{\mathcal{P}}_{1,1} \Longleftrightarrow \alpha \leq 2^{-1}$ and $\mu_{\alpha} \in \mathcal{P}_{1,1} \Longleftrightarrow \alpha<2^{-1}$. Moreover, for the time-dependent measure, given by $\mu=\left\{\left(t^{\frac{1}{2}} \ln \frac{e}{t}\right)^{-1} \delta_{0}: 0 \leq t \leq\right.$ 1 \}, we have $\mu \in \mathcal{P}_{1,1}$ and $\mu \notin \mathcal{P}_{1,1}^{*}$. We refer the reader to Gu3], where similar results were obtained for time-dependent functions. Our next result concerns a time-dependent multiple of the Dirac measure moving along a curve.

Lemma 1. Let $\mu(t)=t^{-\frac{1}{2}} \delta_{\phi(t)}$, where $\phi(t)=t^{\beta}$ with $\beta>0$. Then $\mu \in \mathcal{P}_{1,1}$ if and only if $\beta<\frac{1}{2}$. 
Proof. Let $\beta<\frac{1}{2}$. Then it is easy to see that $\mu \in \hat{\mathcal{P}}_{1,1}$. If $x \leq 0$, then for every $0<t<1$ we have

$$
\begin{array}{r}
\sup _{h: t \leq h \leq 1} \sup _{x \leq 0} \int_{0}^{t} e^{\frac{s}{2} \Delta}|\mu(h-s)|(x) d s \\
=\sup _{h: t \leq h \leq 1} \sup _{x \leq 0} \int_{0}^{t} \frac{1}{\sqrt{2 \pi s} \sqrt{h-s}} \exp \left\{-\frac{\left(x-(h-s)^{\beta}\right)^{2}}{2 s}\right\} d s \\
\leq \int_{0}^{t} \frac{1}{\sqrt{2 \pi s} \sqrt{t-s}} \exp \left\{-\frac{(t-s)^{2 \beta}}{2 s}\right\} d s,
\end{array}
$$

and the last integral in (17) tends to 0 as $t \rightarrow 0+$. Next we get

$$
\begin{array}{r}
\sup _{h: t \leq h \leq 1} \sup _{x: x \geq h^{\beta}} \int_{0}^{t} e^{\frac{s}{2} \Delta}|\mu(h-s)|(x) d s \\
\leq \sup _{h: t \leq h \leq 1} \int_{0}^{t} \frac{1}{\sqrt{2 \pi s} \sqrt{h-s}} \exp \left\{-\frac{\left(h^{\beta}-(h-s)^{\beta}\right)^{2}}{2 s}\right\} d s \\
=\sup _{h: t \leq h \leq 1} \int_{0}^{\frac{t}{h}} \frac{1}{\sqrt{2 \pi u} \sqrt{1-u}} \exp \left\{-\frac{\left(u^{\beta}-(1-u)^{\beta}\right)^{2}}{2 u h^{1-2 \beta}}\right\} d u
\end{array}
$$

$$
\leq \max \left\{\int_{0}^{1} \frac{1}{\sqrt{2 \pi u} \sqrt{1-u}} \exp \left\{-\frac{\left(u^{\beta}-(1-u)^{\beta}\right)^{2}}{2 u t^{\frac{1-2 \beta}{2}}}\right\} d u, \int_{0}^{\sqrt{t}} \frac{1}{\sqrt{2 \pi u} \sqrt{1-u}} d u\right\} \rightarrow 0
$$

as $t \rightarrow 0+$.

Let $0<x<h^{\beta}$. Then $x=(h-\alpha)^{\beta}$ with $0<\alpha<h$. Fix $\epsilon$ and $\lambda$ such that $0<\epsilon<1,0<\lambda<\frac{1}{2}$, and

$$
\tau=\epsilon(1-\beta)+\lambda>1
$$

It follows that

$$
\begin{array}{r}
\sup _{h: t \leq h \leq 1} \sup _{x: 0<x<h^{\beta}} \int_{0}^{t} e^{\frac{s}{2} \Delta}|\mu(h-s)|(x) d s \\
=\sup _{h: t \leq h \leq 1} \sup _{\alpha: 0<\alpha<h} \int_{0}^{t} \frac{1}{\sqrt{2 \pi s} \sqrt{h-s}} \exp \left\{-\frac{\left((h-\alpha)^{\beta}-(h-s)^{\beta}\right)^{2}}{2 s}\right\} d s \\
\quad \leq \max \left\{\sup _{h: t^{\epsilon} \leq h \leq 1} \int_{0}^{t} \frac{1}{\sqrt{2 \pi s} \sqrt{h-s}} d s,\right. \\
\left.\sup _{h: t \leq h \leq t^{\epsilon} \alpha: 0<\alpha<h} \sup _{0} \int_{0}^{t} \frac{1}{\sqrt{2 \pi s} \sqrt{h-s}} \exp \left\{-\frac{\left((h-\alpha)^{\beta}-(h-s)^{\beta}\right)^{2}}{2 s}\right\} d s\right\} \\
=\max \{o(1), I(t)\},
\end{array}
$$

where

$$
I(t)=\sup _{h: t \leq h \leq t^{\epsilon} \alpha: 0<\alpha<h} \sup _{0} \int_{0}^{t} \frac{1}{\sqrt{2 \pi s} \sqrt{h-s}} \exp \left\{-\frac{\left((h-\alpha)^{\beta}-(h-s)^{\beta}\right)^{2}}{2 s}\right\} d s .
$$

Put $G=\left\{s:\left|(h-\alpha)^{\beta}-(h-s)^{\beta}\right| \leq t^{\lambda}\right\}$. Then we have

$$
\begin{aligned}
I(t) \leq \sup _{h: t \leq h \leq t^{\epsilon}} \int_{0}^{t} \frac{1}{\sqrt{2 \pi s} \sqrt{h-s}} \exp \left\{-\frac{t^{2 \lambda}}{2 s}\right\} d s+\sup _{h: t \leq h \leq t^{\epsilon}} \int_{[0, t] \cap G} \frac{1}{\sqrt{2 \pi s} \sqrt{h-s}} d s \\
\\
=I_{1}(t)+I_{2}(t) .
\end{aligned}
$$


It is not difficult to show that

$$
\lim _{t \rightarrow 0+} I_{1}(t)=0 .
$$

It follows from the mean value theorem that $G \subset[0, t] \cap J(\alpha)$, where $J(\alpha)=$ $\left[\alpha-\beta^{-1} t^{\lambda} h^{1-\beta}, \alpha+\beta^{-1} t^{\lambda} h^{1-\beta}\right]$. Therefore, for the small values of $t$ we have

$$
I_{2}(t) \leq \int_{\left[0,2 \beta^{-1} t^{\tau}\right]} \frac{1}{\sqrt{2 \pi s} \sqrt{t-s}} d s,
$$

where $\tau$ is defined by (19). Since $\tau>1$, we have

$$
\lim _{t \rightarrow 0+} I_{2}(t)=0 .
$$

Now (17)-(18) and (20)-(23) give $\mu \in \mathcal{P}_{1,1}$.

If $\beta \geq \frac{1}{2}$, then

$$
\begin{array}{r}
\sup _{h: t \leq h \leq 1} \sup _{x \leq 0} \int_{0}^{t} e^{\frac{s}{2} \Delta}|\mu(h-s)|(x) d s \geq \int_{0}^{t} \frac{1}{\sqrt{2 \pi s} \sqrt{t-s}} \exp \left\{-\frac{(t-s)^{2 \beta}}{2 s}\right\} d s \\
=\int_{0}^{1} \frac{1}{\sqrt{2 \pi u} \sqrt{1-u}} \exp \left\{-\frac{t^{2 \beta-1}(1-u)^{2 \beta}}{2 u}\right\} d u .
\end{array}
$$

Since the last integral in (24) does not tend to 0 as $t \rightarrow 0+$, we get $\mu \notin \mathcal{P}_{1,1}$.

This completes the proof of Lemma 1.

\section{A probabilistic Characterization of Classes OF TIME-DEPENDENT MEASURES}

Let us denote by $\left(\Omega, \Sigma,\left\{\mathcal{F}_{t}\right\},\left\{\theta_{t}\right\}, P_{x}\right)$ the Wiener space, where $\left\{\mathcal{F}_{t}\right\}$ is the standard Brownian filtration, and $\left\{\theta_{t}\right\}$ stands for the family of translations on $\Omega$.

Theorem 2. Let $\mu \in \mathcal{P}_{n, \infty}^{*}$, and assume that $\mu(t) \geq 0$ for all $t \geq 0$. Then there exists a unique (up to equivalence) family $A_{\mu}(t, h), t, h \geq 0$, of random variables on $\Omega$ such that

1. For all $t, h \geq 0$ and $x \in R^{n}$, the function $A_{\mu}(t, h)$ is defined $P_{x}$-a.s.

2. For all $h \geq 0$ and $x \in R^{n}, A_{\mu}(0, h)=0 P_{x}$-a.s.

3. For all $t, h \geq 0, A_{\mu}(t, h)$ is a function of $B_{u}: 0 \leq u \leq t$.

4. For every $h \geq 0$ and $x \in R^{n}$, the function $t \rightarrow A_{\mu}(t, h)$ is non-decreasing and continuous $P_{x}$-a.s.

5. For all $t, \tau, h \geq 0$ and $x \in R^{n}$, the following equality holds $P_{x}$-a.s: $A_{\mu}(t+\tau, h)=A_{\mu}(t, h)+A_{\mu}(\tau, t+h) \circ \theta_{t}$.

6. For all $t, h \geq 0$ and $x \in R^{n}, S_{\mu}(t, h, x)=E_{x} A_{\mu}(t, h)$.

Proof of Theorem 2. Let $V$ be a function on $[0, \infty) \times R^{n}$ such that $V \in \mathcal{P}_{n, \infty}^{*}$. Here we do not assume that the function $V$ is nonnegative. For every $0 \leq t \leq \infty$ and $0 \leq h<\infty$, put

$$
\tilde{A}_{V}(t, h)=\int_{0}^{t} e^{-s} V\left(s+h, B_{s}\right) d s .
$$

Lemma 2. The following estimate holds for $V \in \mathcal{P}_{n, \infty}^{*}$ :

$$
\sup _{h \geq 0} \sup _{x \in R^{n}} E_{x} \tilde{A}_{V}(\infty, h)^{2} \leq 2 \sup _{\lambda \geq 0} \sup _{x \in R^{n}}\left|\tilde{N}_{V}(\infty, \lambda, x)\right| \sup _{\lambda \geq 0} \sup _{x \in R^{n}} \tilde{N}_{|V|}(\infty, \lambda, x) .
$$


Proof. Using the Markov property of the Brownian motion, we get

$$
\begin{aligned}
E_{x} \tilde{A}_{V}(\infty, h)^{2} \\
\quad=2 E_{x} \int_{0}^{\infty} e^{-s} V\left(s+h, B_{s}\right) d s \int_{s}^{\infty} e^{-u} V\left(u+h, B_{u}\right) d u \\
=2 E_{x} \int_{0}^{\infty} e^{-2 s} V\left(s+h, B_{s}\right) d s \int_{0}^{\infty} e^{-\tau} V\left(s+\tau+h, B_{s+\tau}\right) d \tau \\
=2 E_{x} \int_{0}^{\infty} e^{-2 s} d s \int_{0}^{\infty} e^{-\tau} E_{x}\left(V\left(s+h, B_{s}\right) V\left(s+\tau+h, B_{s+\tau}\right) \mid \mathcal{F}_{s}\right) d \tau \\
=2 \int_{0}^{\infty} e^{-2 s} d s E_{x}\left(V\left(s+h, B_{s}\right) \int_{0}^{\infty} e^{-\tau} E_{B_{s}} V\left(s+\tau+h, B_{\tau}\right) d \tau\right) \\
=2 \int_{0}^{\infty} e^{-2 s} E_{x}\left(V\left(s+h, B_{s}\right) d s \int_{0}^{\infty} e^{-\tau} e^{\frac{\tau}{2} \Delta} V(s+\tau+h)\left(B_{s}\right) d \tau\right) \\
=2 \int_{0}^{\infty} e^{-2 s} d s e^{\frac{s}{2} \Delta}\left(V(s+h, x) \int_{0}^{\infty} e^{-\tau} e^{\frac{\tau}{2} \Delta} V(s+\tau+h)(x) d \tau\right) .
\end{aligned}
$$

It follows from (25) that

$$
\sup _{x \in R^{n}} E_{x} \tilde{A}_{V}(\infty, h)^{2} \leq 2 \sup _{x \in R^{n}} \int_{0}^{\infty} e^{-2 s} d s e^{\frac{s}{2} \Delta}\left(|V(s+h)(x)|\left|\tilde{N}_{V}(\infty, s+h, x)\right|\right) .
$$

Now it is easy to see that (26) implies the estimate in Lemma 2.

The proof of Lemma 2 is thus completed.

Using the properties of the conditional expectation and the Markov property, it is not difficult to prove that for all $x \in R^{n}, h \geq 0$, and $t \geq 0$, the following equality holds:

$$
E_{x}\left(\tilde{A}_{V}(\infty, h) \mid \mathcal{F}_{t}\right)=\tilde{A}_{V}(t, h)+e^{-t} \tilde{N}_{V}\left(\infty, t+h, B_{t}\right) .
$$

Lemma 3. We have

$$
\sup _{h \geq 0} \sup _{x \in R^{n}} E_{x} \sup _{t \geq 0} \tilde{A}_{V}(t, h)^{2} \leq 18 \sup _{\lambda \geq 0} \sup _{x \in R^{n}}\left|\tilde{N}_{V}(\infty, \lambda, x)\right| \sup _{\lambda \geq 0} \sup _{x \in R^{n}} \tilde{N}_{|V|}(\infty, \lambda, x) .
$$

Proof. Put

$$
\mathcal{M}_{V}(t, h)=\tilde{A}_{V}(t, h)+e^{-t} \tilde{N}_{V}\left(\infty, t+h, B_{t}\right)
$$

It follows from Theorem 1, Lemma 2, and the continuity property of the Brownian motion that for all $h \geq 0, \mathcal{M}_{V}(t, h)$ is a continuous $\mathcal{F}_{t}$-martingale. Then Doob's inequality gives

$$
E_{x} \sup _{0 \leq t \leq T} \mathcal{M}_{V}(t, h)^{2} \leq 4 E_{x} \mathcal{M}_{V}(T, h)^{2} \leq 4 E_{x} \tilde{A}_{V}(\infty, h)^{2}
$$


for all $T>0$ and $h \geq 0$. Using Lemma 2, we get

$$
\begin{aligned}
E_{x} \sup _{t \geq 0} \tilde{A}_{V}(t, h)^{2} & \leq 2 E_{x} \sup _{t \geq 0} \mathcal{M}_{V}(t, h)^{2}+2 E_{x} \sup _{t \geq 0} \tilde{N}_{V}\left(\infty, t+h, B_{t}\right)^{2} \\
& \leq 8 E_{x} \tilde{A}_{V}(\infty, h)^{2}+2 \sup _{t \geq 0} \sup _{z \in R^{n}} \tilde{N}_{V}(\infty, t+h, z)^{2} \\
& \leq 16 \sup _{\lambda \geq 0} \sup _{x \in R^{n}}\left|\tilde{N}_{V}(\infty, \lambda, x)\right| \sup _{\lambda \geq 0} \sup _{x \in R^{n}} \tilde{N}_{|V|}(\infty, \lambda, x) \\
& +2 \sup _{\lambda \geq 0} \sup _{x \in R^{n}} \tilde{N}_{V}(\infty, \lambda, x)^{2} \\
& \leq 18 \sup _{\lambda \geq 0} \sup _{x \in R^{n}}\left|\tilde{N}_{V}(\infty, \lambda, x)\right| \sup _{\lambda \geq 0} \sup _{x \in R^{n}} \tilde{N}_{|V|}(\infty, \lambda, x) .
\end{aligned}
$$

This completes the proof of Lemma 3 .

Let us continue the proof of Theorem 2. Let $\mu \in \mathcal{P}_{n, \infty}^{*}$ be a nonnegative timedependent measure. Put $g_{k}(h, x)=e^{\frac{1}{2 k} \Delta} \mu(h)(x)$, where $k \geq 1, x \in R^{n}$, and $h \geq 0$. Then for $k \geq l$ we have

$\tilde{N}_{g_{k}}(\infty, \lambda)-\tilde{N}_{g_{l}}(\infty, \lambda)=e^{\frac{1}{2 k} \Delta} \tilde{N}_{\mu}(\infty, \lambda)-\tilde{N}_{\mu}(\infty, \lambda)-\left(e^{\frac{1}{2 l} \Delta} \tilde{N}_{\mu}(\infty, \lambda)-\tilde{N}_{\mu}(\infty, \lambda)\right)$.

Using Theorem 1 and (14), we get

$$
\sup _{\lambda \geq 0} \sup _{x \in R^{n}}\left|\tilde{N}_{g_{k}}(\infty, \lambda, x)-\tilde{N}_{g_{l}}(\infty, \lambda, x)\right| \rightarrow 0
$$

as $k, l \rightarrow \infty$. Since $\mu \in \mathcal{P}_{n, \infty}^{*}$, we obtain

$$
\begin{aligned}
\sup _{\lambda \geq 0} \sup _{x \in R^{n}} \sup _{k, l} \tilde{N}_{\left|g_{k}-g_{l}\right|}(\infty, \lambda, x) & \leq 2 \sup _{\lambda \geq 0} \sup _{x \in R^{n}} \sup _{k} \tilde{N}_{g_{k}}(\infty, \lambda, x) \\
& \leq 2 \sup _{\lambda \geq 0} \sup _{x \in R^{n}} \tilde{N}_{\mu}(\infty, \lambda, x)<\infty .
\end{aligned}
$$

Now Lemma 3, (27), and (28) give

$$
\sup _{h \geq 0} \sup _{x \in R^{n}} E_{x} \sup _{t \geq 0}\left(\tilde{A}_{g_{k}}(t, h)-\tilde{A}_{g_{l}}(t, h)\right)^{2} \rightarrow 0
$$

as $k, l \rightarrow \infty$. It follows from (29) that there exists a random variable $\tilde{A}_{\mu}(t, h)$ on $\Omega$ such that

$$
\lim _{k \rightarrow \infty} \sup _{h \geq 0} \sup _{x \in R^{n}} E_{x} \sup _{t \geq 0}\left|\tilde{A}_{g_{k}}(t, h)-\tilde{A}_{\mu}(t, h)\right|^{2}=0 .
$$

Moreover, (30) implies that for every $h \geq 0$ and $x \in R^{n}$ there exists a subsequence $k_{m}$ such that for $v_{m}=g_{k_{m}}$ we have

$$
\lim _{m \rightarrow \infty}\left|\tilde{A}_{g_{m}}(t, h)-\tilde{A}_{\mu}(t, h)\right|=0
$$

uniformly with respect to $t \geq 0$. It is clear that the functional $\tilde{A}$ satisfies conditions 1-3 in Theorem 2. It also satisfies

$$
\tilde{A}_{\mu}(t+\tau, h)=\tilde{A}_{\mu}(t, h)+e^{-t} \tilde{A}_{\mu}(\tau, t+h) \circ \theta_{t} .
$$

Equality (32) follows from the similar equality for $\tilde{A}_{g_{k}}$ and from (31).

Put

$$
A_{\mu}(t, h)=\int_{0}^{t} e^{s} d \tilde{A}_{\mu}(s, h)
$$


It is not difficult to prove that the functional $A_{\mu}$ satisfies conditions 1-4 in Theorem 2. Using (32) and (33), we see that $A_{\mu}$ also satisfies condition 5. Finally, the equality in condition 6 follows from

$$
\begin{aligned}
E_{x} A_{\mu}(t, h)= & \lim _{m \rightarrow \infty} E_{x} \int_{0}^{t} e^{s} d \tilde{A}_{v_{m}}(s, h)=\lim _{m \rightarrow \infty} E_{x} \int_{0}^{t} v_{m}\left(s+h, B_{s}\right) d s \\
& =\lim _{m \rightarrow \infty} \int_{0}^{t} e^{\frac{s}{2} \Delta} v_{m}(s+h)(x) d x=\int_{0}^{t} e^{\frac{s}{2} \Delta} \mu(s+h)(x) d s .
\end{aligned}
$$

Our next goal is to prove the uniqueness of the functional $A_{\mu}$ in Theorem 2. Suppose that there exist two functionals $A^{1}(t, h)$ and $A^{2}(t, h)$, satisfying all conditions in Theorem 2. Then we have

$$
\begin{array}{r}
E_{x}\left(\int_{0}^{\infty} e^{-s} d A^{1}(s, h)-\int_{0}^{\infty} e^{-s} d A^{2}(s, h)\right)^{2} \\
=2 \Sigma_{i, j=1}^{2} E_{x} \int_{0}^{\infty} e^{-s} d A^{i}(s, h) \int_{s}^{\infty} e^{-u} d A^{j}(u, h) .
\end{array}
$$

Using the Markov property and reasoning as in the proof of Lemma 2, we get that for all $i$ and $j$ with $1 \leq i, j \leq 2$,

$$
\begin{array}{r}
E_{x} \int_{0}^{\infty} e^{-s} d A^{i}(s, h) \int_{s}^{\infty} e^{-u} d A^{j}(u, h) \\
=E_{x} \int_{0}^{\infty} e^{-2 s} d A^{i}(s, h) \int_{0}^{\infty} e^{-u} d A^{j}(u+s, h) \\
=E_{x} \int_{0}^{\infty} e^{-2 s} d A^{i}(s, h) E_{x}\left(\int_{0}^{\infty} e^{-u} d A^{j}(u+s, h) \mid \mathcal{F}_{s}\right) \\
=E_{x} \int_{0}^{\infty} e^{-2 s} d A^{i}(s, h) E_{x}\left(\int_{0}^{\infty} e^{-u} d A^{j}(u, s+h) \circ \theta_{s} \mid \mathcal{F}_{s}\right) \\
=\left.E_{x} \int_{0}^{\infty} e^{-2 s} d A^{i}(s, h) E_{z} \int_{0}^{\infty} e^{-u} d A^{j}(u, s+h) \circ \theta_{s}\right|_{z=B_{s}} \\
=\left.E_{x} \int_{0}^{\infty} e^{-2 s} d A^{i}(s, h) E_{z} \int_{0}^{\infty} e^{-u} d A^{j}(u, s+h ; x+\omega(r): s \leq r \leq u+s)\right|_{z=B_{s}} \\
=\left.E_{x} \int_{0}^{\infty} e^{-2 s} d A^{i}(s, h) E_{z} \int_{0}^{\infty} e^{-u} d A^{j}(u, s+h ; z+\omega(\rho): 0 \leq \rho \leq u)\right|_{z=B_{s}} \\
(35) \quad=E_{x} \int_{0}^{\infty} e^{-2 s} \tilde{N}_{\mu}\left(\infty, h+s, B_{s}\right) d A^{i}(s, h) .
\end{array}
$$

It follows from (34) and (35) that

$$
E_{x}\left(\int_{0}^{\infty} e^{-s} d A^{1}(s, h)-\int_{0}^{\infty} e^{-s} d A^{2}(s, h)\right)^{2}=0 .
$$

Therefore,

$$
\int_{0}^{\infty} e^{-s} d A^{1}(s, h)=\int_{0}^{\infty} e^{-s} d A^{2}(s, h)
$$


a.s. Next, reasoning as in the proof of Lemma 2, we get that for $1 \leq i \leq 2$ and $t>0$,

$$
\begin{aligned}
E_{x}\left(\int_{0}^{\infty} e^{-s} d A^{i}(s, h) \mid \mathcal{F}_{t}\right)= & \int_{0}^{t} e^{-s} d A^{i}(s, h)+E_{x}\left(\int_{t}^{\infty} e^{-s} d A^{i}(s, h) \mid \mathcal{F}_{t}\right) \\
& =\int_{0}^{t} e^{-s} d A^{i}(s, h)+e^{-t} \tilde{N}_{\mu}\left(\infty, t+h, B_{t}\right) .
\end{aligned}
$$

It follows from (36) and (37) that

$$
\int_{0}^{t} e^{-s} d A^{1}(s, h)=\int_{0}^{t} e^{-s} d A^{2}(s, h)
$$

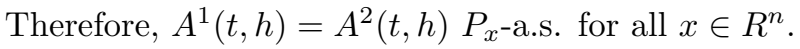

This completes the proof of Theorem 2 .

Definition 4. For any $\mu \in \mathcal{P}_{n, \infty}^{*}$, we put $A_{\mu}(t, h)=A_{\mu^{+}}(t, h)-A_{\mu^{-}}(t, h)$, where $\mu^{+}$and $\mu^{-}$stand for the positive and the negative part of $\mu$, respectively.

Remark 4. Let $\mu \in \mathcal{P}_{n, T}^{*}$. Then the functional $A_{\mu}(t, h)$ is defined for all $0 \leq t \leq T$ and $0 \leq h \leq T-t$ (see the proof of Theorem 2). It is easy to see, using Theorem 2 , that

$$
\lim _{t \rightarrow 0+} \sup _{h: 0 \leq h \leq T-t} \sup _{x \in R^{n}} E_{x} A_{|\mu|}(t, h)=0 .
$$

Lemma 4. Let $\mu \in \mathcal{P}_{n, T}^{*}$. Then

$$
\begin{aligned}
& \quad \sup _{h: 0 \leq h \leq T} \sup _{x \in R^{n}} E_{x} \sup _{t: 0 \leq t \leq T-h} A_{\mu}(t, h)^{2} \\
& \quad \leq c_{T} \sup _{\lambda: 0 \leq \lambda \leq T} \sup _{x \in R^{n}}\left|N_{\mu}(\lambda, x)\right| \sup _{\lambda: 0 \leq \lambda \leq T} \sup _{x \in R^{n}} N_{|\mu|}(\lambda, x),
\end{aligned}
$$

where $c_{T}>0$ is a constant depending only on $T$.

Proof. For every $k \geq 1$ denote $g_{k}(t)=e^{\frac{1}{2 k} \Delta} \mu^{+}(t), q_{k}(t)=e^{\frac{1}{2 k} \Delta} \mu^{-}(t)$. Applying Lemma 3 to $V=g_{k}-q_{k}$, we get

$$
\begin{array}{r}
\sup _{x \in R^{n}} E_{x} \sup _{t: 0 \leq t \leq T-h}\left(\tilde{A}_{g_{k}}(t, h)-\tilde{A}_{q_{k}}(t, h)\right)^{2} \\
\leq 18 \sup _{\lambda: 0 \leq \lambda \leq T} \sup _{x \in R^{n}}\left|N_{g_{k}}(\lambda, x)-N_{q_{k}}(\lambda, x)\right| \sup _{\lambda: 0 \leq \lambda \leq T} \sup _{x \in R^{n}} N_{\left|g_{k}-h_{k}\right|}(\lambda, x)
\end{array}
$$

for every $0 \leq h \leq T$. Now (30) and Theorem 1 give

$$
\begin{array}{r}
\sup _{h: 0 \leq h \leq T} \sup _{x \in R^{n}} E_{x} \sup _{t: 0 \leq t \leq T-h} \tilde{A}_{\mu}(t, h)^{2} \\
\leq 18 \sup _{\lambda: 0 \leq \lambda \leq T} \sup _{x \in R^{n}}\left|N_{\mu}(\lambda, x)\right| \sup _{\lambda: 0 \leq \lambda \leq T} \sup _{x \in R^{n}} N_{|\mu|}(\lambda, x) .
\end{array}
$$

It is clear that

$$
A_{\mu}(t, h)=e^{t} \tilde{A}_{\mu}(t, h)-\int_{0}^{t} \tilde{A}_{\mu}(u, h) e^{u} d u
$$


It follows from (39) that

$$
\begin{aligned}
& \sup _{h: 0 \leq h \leq T} \sup _{x \in R^{n}} E_{x} \sup _{t: 0 \leq t \leq T-h} A_{\mu}(t, h)^{2} \\
& \leq \alpha_{T}\left(\sup _{0 \leq h \leq T} \sup _{x \in R^{n}} E_{x} \sup _{0 \leq t \leq T-h} \tilde{A}_{\mu}(t, h)^{2}\right. \\
& \left.\quad+\sup _{0 \leq h \leq T} \sup _{x \in R^{n}} E_{x} \sup _{0 \leq t \leq T-h} \int_{0}^{t} \tilde{A}_{\mu}(u, h)^{2} d u\right) \\
& \quad \leq \beta_{T} \sup _{0 \leq h \leq T} \sup _{x \in R^{n}} E_{x} \sup _{0 \leq t \leq T-h} \tilde{A}_{\mu}(t, h)^{2} .
\end{aligned}
$$

Now using (38) and (40), we see that the estimate in Lemma 4 holds.

Remark 5. Using (30) and (40), we see that for $\mu \in \mathcal{P}_{n, T}^{*}$,

$$
\lim _{k \rightarrow \infty} \sup _{h: 0 \leq h \leq T} \sup _{x \in R^{n}} E_{x} \sup _{t: 0 \leq t \leq T-h}\left|A_{\mu}(t, h)-\int_{0}^{t} e^{\frac{1}{2 k} \Delta} \mu(s+h)\left(B_{s}\right) d s\right|^{2}=0 .
$$

Definition 5. For $\mu \in \mathcal{P}_{n, T}$, we define the functional $C_{\mu}$ as follows: $C_{\mu}(t, h)=$ $A_{\nu}(t, T-h)$, where $0 \leq t \leq T, t \leq h \leq T$, and $\nu \in \mathcal{P}_{n, T}^{*}$ is given by formula (4).

Remark 6. Let $\mu \in \mathcal{P}_{n, T}$. Then it follows from Remark 4 that

$$
\lim _{t \rightarrow 0+} \sup _{h: t \leq h \leq T} \sup _{x \in R^{n}} E_{x} C_{|\mu|}(t, h)=0 .
$$

It is not difficult to see that the functional $C_{\mu}$ satisfies

$$
C_{\mu}(t+\tau, h)=C_{\mu}(t, h)+C_{\mu}(\tau, h-t) \circ \theta_{t}
$$

for all $0 \leq t \leq h \leq T$ and $0 \leq t+\tau \leq T$.

The next lemma follows from Lemma 4 .

Lemma 5. Let $\mu \in \mathcal{P}_{n, T}$. Then

$$
\begin{array}{r}
\sup _{h: 0 \leq h \leq T} \sup _{x \in R^{n}} E_{x} \sup _{t: 0 \leq t \leq h} C_{\mu}(t, h)^{2} \\
\leq c_{T} \sup _{\lambda: 0 \leq \lambda \leq T} \sup _{x \in R^{n}}\left|M_{\mu}(\lambda, x)\right| \sup _{\lambda: 0 \leq \lambda \leq T} \sup _{x \in R^{n}} M_{|\mu|}(\lambda, x),
\end{array}
$$

where $c_{T}>0$ is a constant depending only on $T$.

Remark 7. For $\mu \in \mathcal{P}_{n, T}$, we have

$$
\lim _{k \rightarrow \infty} \sup _{h: 0 \leq h \leq T} \sup _{x \in R^{n}} E_{x} \sup _{t: 0 \leq t \leq h}\left|C_{\mu}(t, h)-\int_{0}^{t} e^{\frac{1}{2 k} \Delta} \mu(h-s)\left(B_{s}\right) d s\right|^{2}=0 .
$$

\section{Existence of Feynman-Kac propagators and their properties}

In this section, we study the behavior of Feynman-Kac propagators and backward propagators. For Banach spaces $A$ and $B$, we denote by $L(A, B)$ the space of bounded linear operators from $A$ into $B$.

Theorem 3. (a) Let $\mu \in \mathcal{P}_{n, T}$ and $1<p \leq \infty$. Then the family $U_{\mu}$ is a propagator for equation (1).

(b) There exists a time-dependent function $V \in \mathcal{P}_{n, T}$ such that $U_{V}(t, 0) \notin$ $L\left(L^{1}, L^{1}\right)$ for all $0<t \leq T$.

Similarly, if $\mu \in \mathcal{P}_{n, T}^{*}$ and $1<p \leq \infty$, then the family $Y_{\mu}$ is a backward propagator for equation (3). 
Remark 8. It is clear from (2) and (5) that the operators $U_{\mu}(t, \tau)$ with $\mu \in \mathcal{P}_{n, T}$ and $Y_{\mu}(t, \tau)$ with $\mu \in \mathcal{P}_{n, T}^{*}$ are positivity preserving on $L^{p}$ for all $1<p \leq \infty$. Moreover, if $\mu \in \mathcal{P}_{n, T}$, and if $f \in L^{p}$ is a nonnegative function such that $f(x) \neq 0$ on a set of positive measure, then $U_{\mu}(t, \tau) f(x)>0$ for all $0 \leq \tau<t \leq T$ and $x \in R^{n}$. Indeed, it follows from the definition of the class $\mathcal{P}_{n, T}$ that if $U_{\mu}(t, \tau) f(x)=0$ for some $x \in R^{n}$, then $f(x+\omega(t-\tau))=0$ almost everywhere on the Wiener space. Hence, $e^{\frac{t-\tau}{2} \Delta} f(x)=E_{x}\left(f\left(B_{t-\tau}\right)\right)=0$, which is a contradiction. A similar result holds for $Y_{\mu}(t, \tau) f$, where $\mu \in \mathcal{P}_{n, T}^{*}, 0 \leq \tau<t \leq T$, and $f \in L^{p}$ with $1<p \leq \infty$.

Theorem 4. Let $\mu \in \mathcal{P}_{n, T}$. Then $U_{\mu}(t, \tau) \in L\left(L^{p}, L^{q}\right)$ for all $1<p \leq q \leq \infty$ and $0 \leq \tau<t \leq T$. Moreover,

$$
\left\|U_{\mu}(t, \tau)\right\|_{L^{p} \rightarrow L^{q}} \leq A e^{\omega(t-\tau)}(t-\tau)^{-\frac{n}{2}\left(\frac{1}{p}-\frac{1}{q}\right)} .
$$

In (42), the constant $A>0$ is independent of $t, \tau$, and $\mu$, while the constant $\omega=\omega(\mu)$ depends on $\mu$.

Proof of Theorems 3(a) and 4. The proof of these theorems is similar to that of the corresponding results in the case of the heat equation with a time-dependent potential (see [Gu3]). We will need the following lemma, which is Khas'minski's Lemma for time-dependent measures:

Lemma 6. Let $\mu \in \mathcal{P}_{n, T}$, and let $t$ be a number such that $0<t<T$ and

$$
\alpha=\sup _{\delta: 0<\delta \leq t} \sup _{h: \delta \leq h \leq T} \sup _{x \in R^{n}} E_{x} C_{|\mu|}(\delta, h)<1 .
$$

Then

$$
\sup _{h: t \leq h<T} \sup _{x \in R^{n}} E_{x} \exp \left\{C_{|\mu|}(t, h)\right\}<\frac{1}{1-\alpha} .
$$

We refer the reader to $[\underline{S}]$ for the proof of Khas'minski's Lemma for the Kato class potentials and to [Gu3] and [N] for the case of nonautonomous Kato classes of functions. The case of time-independent measures was considered in [BM1, Lemma 2.6. Lemma 6 above can be obtained exactly as Lemma 5 in [Gu3]. It is also possible to use Lemma 5 in Gu3 and Lemma 5 in the present paper to prove Lemma 6.

Remark 9. For $p=q=\infty$, the constant $\omega(\mu)$ in estimate (42) can be described as follows:

$$
\omega(\mu)=c\left(\rho_{\mu}\right)^{-1}
$$

where $c>0$ is an absolute constant and

$$
\rho_{\mu}=\sup \left\{\delta: \sup _{h: \delta \leq h \leq T} \sup _{x \in R^{n}} E_{x} C_{|\mu|}(\delta, h)<\frac{1}{2}\right\} .
$$

This formula can be obtained exactly as estimate (46) in [Gu3]. Similar formula holds in the case $1<p<\infty$ (see (50) in [Gu3]).

Let $\mu \in \mathcal{P}_{n, T}$. It follows from Lemma 6 that for $f \in L^{\infty}$ and every $x \in R^{n}$, the expression $U_{\mu}(t, \tau) f(x)$ is finite, and we have

$$
\left|U_{\mu}(t, \tau) f(x)\right| \leq c e^{\omega(t-\tau)}\|f\|_{\infty} .
$$

Hence, $U_{\mu}(t, \tau) \in L\left(L^{\infty}, L^{\infty}\right)$. For $1<p<\infty$, the boundedness of $U_{\mu}$ in $L^{p}$ with $1<p<\infty$ can be obtained exactly as in the case of absolutely continuous measures (see [Gu3]). Similarly, we get the $\left(L^{p}-L^{q}\right)$-boundedness of $U_{\mu}(t, \tau)$ for 
$1<p \leq q \leq \infty$ and estimate (42) (see [Gu3]). The flow property in part 1 of the definition of a propagator follows from the Markov property of the Brownian motion and from (41). Part 2 easily follows from the definition of $U_{\mu}$.

In the next remark, we will discuss how to define the family $Y_{\mu}$ for $\mu \in \mathcal{P}_{n, T}$. The family $U_{\mu}$ for $\mu \in \mathcal{P}_{n, T}^{*}$ can be defined similarly.

Remark 10. Let $V \in \mathcal{P}_{n, T}$ be a time-dependent function. Then for all $0 \leq \tau \leq t \leq$ $T, x \in R^{n}$, and $P_{0}$-almost all $\omega \in \Omega$ we have

$$
C_{V}(t-\tau, t, x, \omega)=\int_{0}^{t-\tau} V(t-s, x+\omega(s)) d s .
$$

By the continuity of the Brownian motion, the function $(x, \omega) \rightarrow C_{V}(t-\tau, t, x, \omega)$ is measurable. Now let $\mu \in \mathcal{P}_{n, T}$. Then we can find a sequence $V_{k} \in \mathcal{P}_{n, T}$ such that

$$
C_{\mu}(t-\tau, t, x, \omega)=\lim _{k \rightarrow \infty} \int_{0}^{t-\tau} V_{k}(t-s, x+\omega(s)) d s,
$$

where the convergence is in the topology of the space $L_{l o c}^{1}\left(R^{n}\right) \times L^{1}\left(\Omega ; P_{0}\right)$ (see Lemma 5). It follows that the function $(x, \omega) \rightarrow C_{\mu}(t-\tau, t, x, \omega)$ is measurable. Hence for $P_{0}$-almost all $\omega \in \Omega$, the function $x \rightarrow C_{\mu}(t-\tau, t, x-\omega(t-\tau), \omega)$ is Lebesgue measurable on $R^{n}$. Moreover, we have

$$
\begin{array}{r}
C_{\mu}(t-\tau, t, x-\omega(t-\tau), \omega)=\lim _{k \rightarrow \infty} \int_{0}^{t-\tau} V_{k}(t-s, x-\omega(t-\tau)+\omega(s)) d s \\
=\lim _{k \rightarrow \infty} \int_{0}^{t-\tau} V_{k}(\tau+s, x-\omega(t-\tau)+\omega(t-\tau-s)) d s .
\end{array}
$$

Put

$$
Y_{\mu}(t, \tau)=U_{\mu}^{*}(t, \tau)
$$

The operators $Y_{\mu}(t, \tau)$ defined by (43) are bounded on $L^{p}$ with $1 \leq p<\infty$. For all nonnegative functions $g \in L^{p}$ and $f \in L^{p^{\prime}}$, we have

$$
\begin{array}{r}
\int_{R^{n}} g(x) U_{\mu}(t, \tau) f(x) d x \\
=\int_{\Omega} d P_{0}(\omega) \int_{R^{n}} g(x) d x f(x+\omega(t-\tau)) \exp \{-C(t-\tau, t, x, \omega)\} \\
=\int_{\Omega} d P_{0}(\omega) \int_{R^{n}} f(x) d x g(x-\omega(t-\tau)) \exp \{-C(t-\tau, t, x-\omega(t-\tau), \omega)\} \\
=\int_{\Omega} d P_{0}(\omega) \int_{R^{n}} f(x) d x g(x-\omega(t-\tau)) \\
\times \exp \left\{-\lim _{k \rightarrow \infty} \int_{0}^{t-\tau} V_{k}(\tau+s, x-\omega(t-\tau)+\omega(t-\tau-s)) d s\right\} .
\end{array}
$$

By the time-reversibility property of the Brownian motion, $\delta(s)=\omega(t-\tau-s)-\omega(s)$, $0 \leq s \leq t-\tau$, is a standard Brownian motion starting at 0 . It follows from (44) 
that

$$
\begin{aligned}
& =\int_{\Omega} d P_{0}(\delta) \int_{R^{n}} f(x) d x g(x+\delta(t-\tau)) \exp \left\{-\lim _{k \rightarrow \infty} \int_{0}^{t-\tau} V_{k}(\tau+s, x+\delta(s)) d s\right\} \\
& =\int_{R^{n}} f(x) d x \int_{\Omega} d P_{0}(\delta) g(x+\delta(t-\tau)) \exp \left\{-\lim _{k \rightarrow \infty} \int_{0}^{t-\tau} V_{k}(\tau+s, x+\delta(s)) d s\right\} .
\end{aligned}
$$

Put

$$
A_{\mu}(t-\tau, \tau, x, \delta)=\lim _{k \rightarrow \infty} \int_{0}^{t-\tau} V_{k}(\tau+s, x+\delta(s)) d s .
$$

Then $(x, \delta) \rightarrow A_{\mu}(t-\tau, \tau, x, \delta)$ is a measurable function, and it follows from (45) that

$$
Y_{\mu}(t, \tau) g(x)=E_{x} g\left(B_{t-\tau}\right) \exp \left\{-A_{\mu}(t-\tau, \tau)\right\}
$$

for all $g \in L^{p}$. Note that formula (46) is consistent with formula (5), since for $\mu \in \mathcal{P}_{n, T} \cap \mathcal{P}_{n, T}^{*}$, both formulas define the same family of operators.

Combining Theorem 3 and a similar result for the class $\mathcal{P}_{n, T}^{*}$, we get the following theorem:

Theorem 5. Let $\mu \in \mathcal{P}_{n, T} \cap \mathcal{P}_{n, T}^{*}$ and $1 \leq p \leq \infty$. Then:

1. The family $U_{\mu}$ is a propagator for equation (1).

2. The family $Y_{\mu}$ is a backward propagator for equation (3).

3. The operators $U_{\mu}(t, \tau)$ and $Y_{\mu}(t, \tau)$ satisfy estimate (42) for all $1 \leq p \leq$ $q \leq \infty$.

Our next goal is to prove the joint continuity condition for $U_{\mu}(t, \tau)$ (see part 3 of the definition of a propagator). The following lemma holds:

Lemma 7. Let $\mu \in \mathcal{P}_{n, T}$ and $1<p \leq \infty$. Then

$$
\left\|U_{\mu}(t, \tau)-e^{(t-\tau) \frac{1}{2} \Delta}\right\|_{p \rightarrow p} \rightarrow 0
$$

as $t-\tau \rightarrow 0$.

For a time-dependent function $V \in \mathcal{P}_{n, T}$, Lemma 7 was obtained in [Gu3]. The proof of Lemma 7 in the case of time-dependent measures is similar.

Remark 11. It follows from (43) and (47) that for $\mu \in \mathcal{P}_{n, T}$ and $1 \leq p<\infty$,

$$
\left\|Y_{\mu}(t, \tau)-e^{(t-\tau) \frac{1}{2} \Delta}\right\|_{p \rightarrow p} \rightarrow 0
$$

as $t-\tau \rightarrow 0$.

Let $1<p<\infty$. Then reasoning as in the proof of the joint continuity of $U_{V}$ in Gu3., we can show the strong continuity from the right of the function $t \rightarrow U_{\mu}(t, \tau)$ on the interval $[\tau, T]$ and the strong continuity of the function $\tau \rightarrow U_{\mu}(t, \tau)$ on the interval $[\tau, T]$. Next we will prove that

$$
\lim _{\lambda \rightarrow 0+}\left\|U_{\mu}(t, \tau) f-U_{\mu}(t-\lambda, \tau+\lambda) f\right\|_{p}=0,
$$


where $0 \leq \tau<t \leq T$. Indeed, let $\tau+\lambda \leq t-\lambda$, and choose $\rho$ such that $\rho \leq t-\lambda \leq t$. Then we have

$$
\begin{array}{r}
\left\|U_{\mu}(t, \tau) f-U_{\mu}(t-\lambda, \tau+\lambda) f\right\|_{p} \leq\left\|U_{\mu}(t, \tau) f-U_{\mu}(t, \tau+\lambda) f\right\|_{p} \\
+\|(U(t, \rho)-U(t-\lambda, \rho)) U(\rho, \tau+\lambda) f\|_{p} \leq\|U(t, \tau) f-U(t, \tau+\lambda) f\|_{p} \\
+\left\|\left(e^{\frac{t-\rho}{2} \Delta}-e^{\frac{t-\lambda-\rho}{2} \Delta}\right) U_{\mu}(\rho, \tau+\lambda) f\right\|_{p}+\left\|\left(U_{\mu}(t, \rho)-e^{\frac{t-\rho}{2} \Delta}\right) U_{\mu}(\rho, \tau+\lambda) f\right\|_{p} \\
+\left\|\left(U_{\mu}(t-\lambda, \rho)-e^{\frac{t-\lambda-\rho}{2} \Delta}\right) U_{\mu}(\rho, \tau+\lambda) f\right\|_{p} \leq\left\|U_{\mu}(t, \tau) f-U_{\mu}(t, \tau+\lambda) f\right\|_{p} \\
+M\left\|U_{\mu}(t, \rho)-e^{\frac{t-\rho}{2} \Delta}\right\|_{p \rightarrow p}+M\left\|U_{\mu}(t-\lambda, \rho)-e^{\frac{t-\lambda-\rho}{2}}\right\|_{p \rightarrow p} \\
+M\left\|\left(e^{\frac{\lambda}{2} \Delta}-I\right) U_{\mu}(\rho, \tau+\lambda) f\right\|_{p}=I_{1}+I_{2}+I_{3}+I_{4} .
\end{array}
$$

For a given $\epsilon>0$, fix $\rho$ so close to $t$ that $I_{2}+I_{3} \leq \frac{\epsilon}{3}$. This can be done using (47). Then the continuity of the function $\tau \rightarrow U(t, \tau) f$ implies that there exists $\delta>0$ such that if $0 \leq \lambda \leq \delta$, then $I_{1} \leq \frac{\epsilon}{3}$. Moreover, since the set $\left\{U_{\mu}(\rho, \tau+\lambda) f: 0 \leq\right.$ $\lambda \leq \tau-\rho\}$, is compact in $L^{p}$ and the semigroup $e^{\frac{t}{2} \Delta}$ is strongly continuous in $L^{p}$, we get $I_{4} \leq \frac{\epsilon}{3}$ for $0 \leq \lambda \leq \delta_{1}$. This proves equality (48) for $1<p<\infty$. It is not difficult to see that the remaining cases in the proof of the joint continuity of the function $(t, \tau) \rightarrow U_{\mu}(t, \tau) f$ are similar. The proof in the case $p=\infty$ is also similar.

Remark 12. Let $\mu \in \mathcal{P}_{n, T}$. Then it follows from (43) that for $1 \leq p<\infty$, we have $Y_{\mu} \in L\left(L^{p}, L^{p}\right)$. Moreover, $Y_{\mu}(t, \tau)=Y_{\mu}(\lambda, \tau) Y_{\mu}(t, \lambda)$ for $0 \leq \tau \leq \lambda \leq t \leq T$, and $Y_{\mu}(t, t)=I$ for $0 \leq t \leq T$. The family $Y_{\mu}$ is jointly strongly continuous in $L^{p}$. The proof of this fact is similar to that of the joint continuity of $U_{\mu}$.

It remains to prove that condition 4 in the definition of a propagator holds for $U_{\mu}$. The proof will be given at the end of the present section. First we are going to study the behavior of Feynman-Kac propagators in various spaces of continuous functions.

The next lemma contains a useful estimate for the difference of two Feynman-Kac propagators.

Lemma 8. Let $\mu_{1}, \mu_{2} \in \mathcal{P}_{n, T}$. Then for every $f \in L^{\infty}$ and $0 \leq \tau \leq t \leq T$ we have

$$
\begin{array}{r}
\sup _{x \in R^{n}}\left|U_{\mu_{1}}(t, \tau) f(x)-U_{\mu_{2}}(t, \tau) f(x)\right| \leq \beta \exp \{\zeta(t-\tau)\} \\
\times\left\{\sup _{x \in R^{n}} \sup _{\lambda: 0 \leq \lambda \leq T}\left|M_{\mu_{1}-\mu_{2}}(\lambda, x)\right| \sup _{x \in R^{n}} \sup _{\lambda: 0 \leq \lambda \leq T} M_{\left|\mu_{1}-\mu_{2}\right|}(\lambda, x)\right\}^{\frac{1}{4}}|| f \|_{\infty},
\end{array}
$$

where $\beta>0$ is an absolute constant, and the constant $\zeta$ depends only on $\mu_{1}$ and $\mu_{2}$.

Remark 13. The constant $\zeta$ in Lemma 8 is given by $\zeta=c_{1} \omega\left(c_{2}\left(\left|\mu_{1}\right|+\left|\mu_{2}\right|\right)\right)$, where $c_{1}>0$ and $c_{2}>0$ are absolute constants, and $\omega$ is the constant, defined in Remark 9 and corresponding to the family of measures $c_{2}\left(\left|\mu_{1}\right|+\left|\mu_{2}\right|\right)$. 
Proof of Lemma 8. Using Hölder's inequality, estimate (42), and inequality $e^{y}-1 \leq$ $y e^{y}, y \geq 0$, we get

$$
\begin{aligned}
\mid U_{\mu_{1}}( & t, \tau) f(x)-U_{\mu_{2}}(t, \tau) f(x) \mid \\
\leq & E_{x}\left|\exp \left\{-C_{\mu_{1}}(t-\tau, t)\right\}-\exp \left\{-C_{\mu_{2}}(t-\tau, t)\right\}\right| \mid f \|_{\infty} \\
\leq & \left\{E_{x} \exp \left\{-2 C_{\mu_{1}}(t-\tau, t)\right\}\right\}^{\frac{1}{2}}\left\{E_{x} \exp \left\{2\left|C_{\mu_{1}-\mu_{2}}(t-\tau, t)\right|\right\}-1\right\}^{\frac{1}{2}}\|f\|_{\infty} \\
\leq & \alpha_{1} \exp \left\{2^{-1}(t-\tau) \omega\left(2\left|\mu_{1}\right|\right)\right\}\left\{E_{x} 2\left|C_{\mu_{1}-\mu_{2}}(t-\tau, t)\right|\right. \\
& \left.\times \exp \left\{2\left|C_{\mu_{1}-\mu_{2}}(t-\tau, t)\right|\right\}\right\}^{\frac{1}{2}}\|f\|_{\infty} \leq \alpha_{2} \exp \left\{2^{-1}(t-\tau) \omega\left(2\left|\mu_{1}\right|\right)\right\} \\
& \times\left\{E_{x}\left(C_{\mu_{1}-\mu_{2}}(t-\tau, t)\right)^{2}\right\}^{\frac{1}{4}}\left\{E_{x} \exp \left\{4\left|C_{\mu_{1}-\mu_{2}}(t-\tau, t)\right|\right\}^{\frac{1}{4}}|| f \|_{\infty}\right. \\
\leq & \alpha \exp \left\{c_{1}(t-\tau) \omega\left(c_{2}\left(\left|\mu_{1}\right|+\left|\mu_{2}\right|\right)\right)\right\}\left\{E_{x}\left(\left.C_{\mu_{1}-\mu_{2}}(t-\tau, t)\right|^{2}\right\}^{\frac{1}{4}}\|f\|_{\infty}\right.
\end{aligned}
$$

for every $x \in R^{n}$. Now Lemma 8 follows from (49) and Lemma 5 .

Theorem 6. Let $\mu \in \mathcal{P}_{n, T}$ and $1<p \leq \infty$. Then $U_{\mu}(t, \tau) \in L\left(L^{p}, B C\right)$ for all $0 \leq \tau<t \leq T$.

The next assertion follows from Theorem 6 , a similar theorem for $Y_{\mu}$ with $\mu \in$ $\mathcal{P}_{n, T}^{*}$, and from the joint continuity properties of $U_{\mu}$ and $Y_{\mu}$.

Corollary 1. (a) Let $\mu \in \mathcal{P}_{n, T}$. Then for every $f \in L^{p}$ with $1<p \leq \infty$ and $\tau \in[0, T)$, the function $(t, x) \rightarrow U_{\mu}(t, \tau) f(x)$ is continuous on $(\tau, T) \times R^{n}$.

(b) Let $\mu \in \mathcal{P}_{n, T}^{*}$. Then for every $f \in L^{p}$ with $1<p \leq \infty$ and $0<t \leq T$, the function $(\tau, x) \rightarrow Y_{\mu}(t, \tau) f(x)$ is continuous on $(0, t) \times R^{n}$.

Theorem 7. Let $\mu \in \mathcal{P}_{n, T}$. Then $U_{\mu}(t, \tau) \in L(B U C, B U C)$ and $U_{\mu}(t, \tau) \in$ $L\left(C_{\infty}, C_{\infty}\right)$ for all $0 \leq \tau \leq t \leq T$.

Remark 14. The property $U_{\mu}(t, \tau) \in L\left(C_{\infty}, C_{\infty}\right)$ in Theorem 7 is called the Feller property of the Feynman-Kac propagator $U_{\mu}$. We refer the reader to $\mathrm{DvC}$ for various results concerning the Feller property for semigroups.

Proof of Theorem 6. Let $\mu \in \mathcal{P}_{n, T}$. With no loss of generality we may assume $p=\infty$, since $U_{\mu}(t, \tau) \in L\left(L^{p}, L^{\infty}\right)$, and $U_{\mu}$ satisfies property 1 in the definition of an evolution family. For every $h \in R^{n}$ and $f \in L^{\infty}$, denote by $f_{h}$ the function given by $f_{h}(x)=f(x+h)$, and for a Borel measure $\mu$ denote by $\mu_{h}$ the measure defined by $\mu_{h}(E)=\mu(E+h)$ for all $E \in \mathcal{B}\left(R^{n}\right)$. Since $U_{\mu}(t, \tau) f(x+z)=U_{\mu_{z}}(t, \tau) f_{z}(x)$ for all $x, z \in R^{n}$, we have

$$
\begin{aligned}
\left|U_{\mu}(t, \tau) f(x+z)-U_{\mu}(t, \tau) f(x)\right| & =\left|U_{\mu_{z}}(t, \tau) f_{z}(x)-U_{\mu}(t, \tau) f_{z}(x)\right| \\
& +\left|U_{\mu}(t, \tau)\left(f_{z}-f\right)(x)\right|=I_{1}+I_{2}
\end{aligned}
$$

It follows from Lemma 8 that

$$
\begin{array}{r}
\sup _{x \in R^{n}} I_{1} \leq c\|f\|_{\infty} \sup _{\lambda: 0 \leq \lambda \leq T} \sup _{x \in R^{n}}\left|M_{\mu-\mu_{z}}(\lambda, x)\right|^{\frac{1}{4}} \\
\leq c\|f\|_{\infty}\left\{\sup _{\lambda: 0 \leq \lambda \leq T} \sup _{x \in R^{n}}\left|M_{\mu^{+}}(\lambda, x)-M_{\mu^{+}}(\lambda, x+z)\right|^{\frac{1}{4}}\right. \\
\left.+\sup _{\lambda: 0 \leq \lambda \leq T} \sup _{x \in R^{n}}\left|M_{\mu^{-}}(\lambda, x)-M_{\mu^{-}}(\lambda, x+z)\right|^{\frac{1}{4}}\right\} .
\end{array}
$$


Applying Theorem 1 and taking into account that $\mu^{+}, \mu^{-} \in \mathcal{P}_{n, T}$, we get

$$
\lim _{z \rightarrow 0} \sup _{x \in R^{n}} I_{1}=0 .
$$

Denote by $\chi_{r}$ the characteristic function of the ball of radius $r$ centered at the origin. Then

$$
I_{2} \leq\left|U_{\mu}(t, \tau)\left(f_{z}-f\right) \chi_{r}(x)\right|+\left|U_{\mu}(t, \tau)\left(f_{z}-f\right)\left(1-\chi_{r}\right)(x)\right|=J_{1}+J_{2} .
$$

We have

$$
\sup _{x \in R^{n}} J_{1} \leq c\left\|\left(f_{z}-f\right) \chi_{r}\right\|_{\infty} \leq c_{r}\left\|\left(f_{z}-f\right) \chi_{r}\right\|_{2} .
$$

Hence, for every fixed $r$ we have

$$
\lim _{z \rightarrow 0} \sup _{x \in R^{n}} J_{1}=0 .
$$

It follows from the definition of $U_{\mu}$ that

$$
\begin{array}{r}
\sup _{z:|z| \leq 1} J_{2} \leq 2 \sup _{z:|z| \leq 1} E_{x} f_{z}\left(B_{t-\tau}\right)\left(1-\chi_{r}\right)\left(B_{t-\tau}\right) \exp \left\{-C_{\mu}(t-\tau, t)\right\} \\
\leq 2\|f\|_{\infty} E_{x}\left(1-\chi_{r}\right)\left(B_{t-\tau}\right) \exp \left\{C_{|\mu|}(t-\tau, t)\right\}
\end{array}
$$

for all $x \in R^{n}$. Using the dominated convergence theorem we obtain

$$
\lim _{r \rightarrow \infty} \sup _{z:|z| \leq 1} J_{2}=0 .
$$

Indeed, the pointwise convergence follows from the following fact:

$$
\lim _{r \rightarrow \infty}\left(1-\chi_{r}\right)(x+\omega(t-\tau))=0
$$

for all $x \in R^{n}, 0 \leq \tau<t \leq T$, and almost all $\omega$. Moreover, by Lemma 6 , we have the following estimate:

$$
\sup _{x \in R^{n}} E_{x} \exp \left\{C_{|\mu|}(t-\tau, t)\right\}<\infty .
$$

Using (52), (53), and (54), we see that

$$
\lim _{z \rightarrow 0} I_{2}=0 .
$$

Now it is clear that Theorem 6 follows from (50), (51), and (55).

Proof of Theorem 7. Let $f \in B U C$. Then using the same notation as in (50), we see that (51) holds. Moreover

$$
\sup _{x \in R^{n}} I_{2} \leq c\left\|f_{z}-f\right\|_{\infty},
$$

and therefore,

$$
\lim _{z \rightarrow 0} \sup _{x \in R^{n}} I_{2}=0 .
$$

Now (50), (51), and (56) imply Theorem 7 for the space $B U C$.

Next let $f \in C_{\infty}$. Then it follows from Theorem 6 that the function $U_{\mu}(t, \tau)$ is continuous. Moreover,

$$
\sup _{x \in R^{n}}\left|U_{\mu}(t, \tau) f\left(1-\chi_{r}\right)(x)\right| \leq c|| f\left(1-\chi_{r}\right) \|_{\infty} \rightarrow 0
$$


as $r \rightarrow \infty$. Therefore, for every $x \in R^{n}$ and for a fixed $r>0$ we have

$$
\begin{aligned}
\left|U_{\mu}(t, \tau) f \chi_{r}(x)\right| & \leq c E_{x} \chi_{r}\left(B_{t-\tau}\right) \exp \left\{C_{|\mu|}(t-\tau, t)\right\}\|f\|_{\infty} \\
& \leq c\left\{E_{x} \chi_{r}\left(B_{t-\tau}\right)\right\}^{\frac{1}{2}}\left\{E_{x} \exp \left\{2 C_{|\mu|}(t-\tau, t)\right\}\right\}^{\frac{1}{2}}\|f\|_{\infty} \\
& \leq c\left\{E_{x} \chi_{r}\left(B_{t-\tau}\right)\right\}^{\frac{1}{2}}\|f\|_{\infty}=c e^{\frac{t-\tau}{2} \Delta} \chi_{r}(x)\|f\|_{\infty} .
\end{aligned}
$$

It follows from (58) that

$$
\lim _{x \rightarrow \infty}\left|U_{\mu}(t, \tau) f \chi_{r}(x)\right|=0 .
$$

Next using (57) and (59), we see that Theorem 7 holds for the space $C_{\infty}$.

This completes the proof of Theorem 7 .

Let us return to the proof of Theorem 3. We will show that condition 4 in the definition of a propagator holds for $U_{\mu}$ in the case $p=\infty$. The case $1<p<\infty$ is similar. Put $g_{k}(h)=e^{\frac{1}{2 k} \Delta} \mu(h)$. Since $\mu \in \mathcal{P}_{n, T}$, we have $g_{k} \in \mathcal{P}_{n, T}$ for all $k \geq 1$. Moreover,

$$
\sup _{k} \omega\left(\left|g_{k}\right|\right)<\infty
$$

It follows from Theorem 1 in $\mathrm{Gu} 2$ that $U_{g_{k}}$ satisfies

$$
\begin{aligned}
-\int_{\tau}^{T} \int_{R^{n}} U_{g_{k}}(t, \tau) f(x) \frac{\partial \phi}{\partial t}(t, x) d x d t & =\frac{1}{2} \int_{\tau}^{T} \int_{R^{n}} U_{g_{k}}(t, \tau) f(x) \Delta \phi(t, x) d x d t \\
& -\int_{\tau}^{T} \int_{R^{n}} g_{k}(t, x) U_{g_{k}}(t, \tau) f(x) \phi(t, x) d x d t
\end{aligned}
$$

for all $\phi \in C_{0}^{\infty}\left((\tau, T) \times R^{n}\right)$ and $1<p \leq \infty$. Using (60), Lemma 8, and theorems 1 and 6 we obtain that for all $0 \leq \tau \leq t \leq T$,

$$
\lim _{k \rightarrow \infty} U_{g_{k}}(t, \tau) f=U_{\mu}(t, \tau) f
$$

in the space $B C\left(R^{n}\right)$. We will also need the following inequality:

$$
\sup _{x \in R^{n}} \int_{0}^{T-\epsilon}|\mu(t)|(B(x, r)) d t<\infty
$$

for every $r>0, \epsilon$ such that $0<\epsilon<T$, and $\mu \in \mathcal{P}_{n, T}$. Inequality (63) was obtained in Gu1], Lemma 4, in the case of absolutely continuous measures. The general case is similar. Using (60), (62), (63), the continuity of the functions $U_{g_{k}}(t, \tau) f$ and $U_{\mu}(t, \tau)$, and the dominated convergence theorem, we see from (61), that condition 4 holds.

This completes the proof of theorem 3(a).

Proof of Theorem 3(b). We restrict ourselves to the case $n=3, T=1$. Let us consider the following time-dependent function:

$$
V(t, x)=-\frac{1}{\sqrt{t} \ln \frac{e}{t}|x|},
$$

where $(t, x) \in[0,1] \times R^{3}$. It was shown in [Gu3 that $V \in \mathcal{P}_{3,1}$. Now suppose $U_{V}(t, 0) \in L\left(L^{1}, L^{1}\right)$ for some $t>0$. It follows from Remark 10 that $Y_{V}(t, 0)=$ $U_{V}^{*}(t, 0) \in L\left(L^{\infty}, L^{\infty}\right)$. Hence, the function

$$
x \rightarrow E_{x} \exp \left\{\int_{0}^{t} \frac{d u}{\sqrt{u} \ln \frac{e}{u}\left|B_{u}\right|}\right\}
$$


belongs to the space $L^{\infty}$. This implies $\phi \in L^{\infty}$, where

$$
\phi(x)=E_{x} \int_{0}^{t} \frac{d u}{\sqrt{u} \ln \frac{e}{u}\left|B_{u}\right|}=\int_{0}^{t} \frac{d u}{\sqrt{u} \ln \frac{e}{u}} \frac{1}{(2 \pi u)^{3 / 2}} \int_{R^{n}} \frac{1}{|y|} e^{-\frac{|x-y|^{2}}{2 u}} d y .
$$

Using Fatou's Lemma, we get $\phi(0)<\infty$. On the other hand,

$$
\phi(0)=c \int_{0}^{t} \frac{d u}{u \ln \frac{e}{u}}=\infty .
$$

This contradiction shows that $U_{V} \notin L\left(L^{1}, L^{1}\right)$.

This completes the proof of Theorem $3(\mathrm{~b})$ for $n=3$ and $T=1$. The rest of the cases is similar.

\section{UNIQUENESS OF EXPONENTIALLY BOUNDED SOLUTIONS}

Our first result in this section is a uniqueness theorem for an exponentially bounded distributional solution to equation (1).

Theorem 8. Let $\mu \in \mathcal{P}_{n, T}, 0 \leq \tau<T$, and let $f$ be a Lebesgue measurable function on $R^{n}$. Suppose $u_{1}$ and $u_{2}$ are measurable functions on $[\tau, T] \times R^{n}$ such that for $i=1,2$ the following conditions hold:

1. $\left|u_{i}(t, x)\right| \leq M \exp \{a|x|\}$ for all $(t, x) \in[\tau, T] \times R^{n}$, where $M>0$ and $a>0$ are absolute constants.

2. The function $t \rightarrow u_{i}(t, \cdot)$ is a solution to equation (1) in the $D^{\prime}\left((\tau, T) \times R^{n}\right)$ sense.

3. The function $t \rightarrow u_{i}(t, \cdot)$ is continuous in measure on the interval $[\tau, T]$.

4. $u_{i}(0, x)=f$ a.e. on $R^{n}$.

Then for every $t$ with $\tau \leq t \leq T$ we have $u_{1}(t)=u_{2}(t)$ a.e. on $R^{n}$.

Proof. With no loss of generality we may assume that $\tau=0, f=0, u_{1}=u$, and $u_{2}=0$. We will prove Theorem 8 for $M=a=1$. The general case is similar. Suppose that

$$
\sup _{t: 0 \leq t \leq T} \sup _{x \in R^{n}}|u(t, x)| e^{-|x|}<\infty .
$$

Let $0<t<T, \gamma \in C_{0}^{\infty}(0, t), \phi \in C_{0}^{\infty}\left(R^{n}\right)$, and fix an infinitely differentiable function $\Lambda$ on $R^{n}$ for which $c_{1} e^{-|x|} \leq \Lambda(x) \leq c_{2} e^{-|x|}, \Delta \Lambda(x) \leq c_{3} e^{-|x|}$, and $|\nabla \Lambda(x)| \leq c_{4} e^{-|x|}$. Put $g(s, x)=\gamma(s) \Lambda(x) e^{\frac{t-s}{2} \Delta} \phi(x)$, where $(s, x) \in[0, t] \times R^{n}$, and fix a nonnegative even function $\psi \in C_{0}^{\infty}\left(R^{1}\right)$ such that $\psi$ is nonincreasing on $[0, \infty)$, and $\psi(t)=1$ for all $t \in R^{1}$ with $|t| \leq 1$. Then $\psi_{k} g \in C_{0}^{\infty}\left((0, t) \times R^{n}\right)$, where we put $\psi_{k}(x)=\psi\left(k^{-1}|x|\right)$ for all $k \geq 1$ and $x \in R^{n}$. It follows from the definition of a distributional solution that

$$
\begin{aligned}
-\int_{0}^{t} & \int_{R^{n}} u(s) \frac{\partial\left(\psi_{k} g\right)}{\partial s} d x d s \\
& =\frac{1}{2} \int_{0}^{t} \int_{R^{n}} u(s) \Delta\left(\psi_{k} g\right) d x d s-\int_{0}^{t} \int_{R^{n}} u(s) \psi_{k} g(s) d \mu(s) d s .
\end{aligned}
$$

Using the dominated convergence theorem in the previous equality, we get

$$
-\int_{0}^{t} \int_{R^{n}} u(s) \frac{\partial g(s)}{\partial s} d x d s=\frac{1}{2} \int_{0}^{t} \int_{R^{n}} u(s) \Delta g(s) d x d s-\int_{0}^{t} \int_{R^{n}} u(s) g(s) d \mu(s) d s .
$$


In the proof of equality (65) we used the following estimate:

$$
\int_{0}^{t} \int_{R^{n}}|u(s)| \Lambda e^{\frac{t-s}{2} \Delta}|\phi| d|\mu(s)| d s \leq c \int_{0}^{t} \int_{R^{n}} e^{\frac{t-s}{2} \Delta}|\mu(s)| d x d s<\infty .
$$

Estimate (66) holds, since $\mu \in \mathcal{P}_{n, T}$. It follows from (65) that

$$
\begin{array}{r}
\int_{0}^{t} \gamma^{\prime}(s) d s \int_{R^{n}} u(s) \Lambda e^{\frac{t-s}{2} \Delta} \phi d x=\int_{0}^{t} \gamma(s) d s \int_{R^{n}} u(s) \Lambda e^{\frac{t-s}{2} \Delta} \phi d \mu(s) \\
-\frac{1}{2} \int_{0}^{t} \gamma(s) d s \int_{R^{n}} u(s)(\Delta \Lambda) e^{\frac{t-s}{2} \Delta} \phi d x-\int_{0}^{t} \gamma(s) d s \int_{R^{n}} u(s)\left(\nabla \Lambda \cdot \nabla e^{\frac{t-s}{2} \Delta} \phi\right) d x .
\end{array}
$$

Let us denote

and

$$
\eta(s)=\int_{R^{n}} u(s) \Lambda e^{\frac{t-s}{2} \Delta} \phi d x
$$

$$
\begin{aligned}
\zeta(s)= & \int_{R^{n}} u(s) \Lambda e^{\frac{t-s}{2} \Delta} \phi d \mu(s)-\frac{1}{2} \int_{R^{n}} u(s)(\Delta \Lambda) e^{\frac{t-s}{2} \Delta} \phi d x \\
& -\int_{R^{n}} u(s)\left(\nabla \Lambda \cdot \nabla e^{\frac{t-s}{2} \Delta} \phi\right) d x .
\end{aligned}
$$

Then we have $\zeta \in L^{1}[0, t]$. Hence, $\eta \in W_{1}^{1}(0, t)$, and $\frac{\partial}{\partial s} \eta(s)=\zeta(s)$ almost everywhere on $(0, t)$. Therefore, there exists a decreasing sequence $\epsilon_{k} \rightarrow 0$ such that

$$
\begin{array}{r}
\int_{R^{n}} u\left(t-\epsilon_{k}, x\right) \Lambda(x) e^{\frac{\epsilon_{k}}{2} \Delta} \phi(x) d x-\int_{R^{n}} u\left(\epsilon_{k}, x\right) \Lambda(x) e^{\frac{t-\epsilon_{k}}{2} \Delta} \phi(x) d x \\
=\int_{R^{n}} \phi(x) d x\left(\int _ { \epsilon _ { k } } ^ { t - \epsilon _ { k } } \left[e^{\frac{t-s}{2} \Delta}(u(s) \Lambda \mu(s))(x)-\frac{1}{2} e^{\frac{t-s}{2} \Delta}(u(s) \Delta \Lambda)(x)\right.\right. \\
\left.\left.+\sum_{i=1}^{n} \frac{\partial}{\partial x_{i}} e^{\frac{t-s}{2} \Delta}\left(u(s) \frac{\partial}{\partial x_{i}} \Lambda\right)(x)\right] d s\right) .
\end{array}
$$

Using the dominated convergence theorem, the assumptions in Theorem 8, and the properties of the heat semigroup, we see that the equality

$$
\begin{array}{r}
u(t, x) \Lambda(x)=\int_{0}^{t}\left[e^{\frac{t-s}{2} \Delta}(u(s)\right. \\
\Lambda \mu(s))(x)-\frac{1}{2} e^{\frac{t-s}{2} \Delta}(u(s) \Delta \Lambda)(x) \\
\left.+\sum_{i=1}^{n} \frac{\partial}{\partial x_{i}} e^{\frac{t-s}{2} \Delta}\left(u(s) \frac{\partial}{\partial x_{i}} \Lambda\right)(x)\right] d s
\end{array}
$$

holds in the space $D^{\prime}\left((0, t) \times R^{n}\right)$. It follows from the properties of the function $\Lambda$ and from the condition $\mu \in \mathcal{P}_{n, T}$ that the function on the right-hand side of (67) belongs to the space $L^{\infty}\left((0, T) \times R^{n}\right)$. Hence, equality (67) holds in the space $L^{\infty}\left((0, t) \times R^{n}\right)$. Now $(67)$ implies that for every $t$ with $0<t<T$ we have

$$
\begin{aligned}
& \sup _{s: 0 \leq s \leq t} \sup _{x \in R^{n}}\left\{|u(s, x)| e^{-|x|}\right\} \\
& \quad \leq c \sup _{s: 0 \leq s \leq t} \sup _{x \in R^{n}}\left\{|u(s, x)| e^{-|x|}\right\}\left(\sup _{x} \int_{0}^{t} e^{\frac{t-s}{2}}|\mu(s)|(x) d s+t+\sqrt{t}\right),
\end{aligned}
$$

where $c>0$ is independent of $t$. Since $\mu \in \mathcal{P}_{n, T}$, we get $u(s, \cdot)=0$ a.e. on $R^{n}$ for all $s$ with $0 \leq s \leq t_{0}$. Using the same reasoning, we can extend the previous local result to the global equality $u(s)=0$ a.e. for all $0 \leq s \leq T$.

This completes the proof of Theorem 8 . 
Remark 15. We can replace the convergence in measure in condition 3 in Theorem 8 by the convergence almost everywhere. We can also use the following condition instead of condition 3: For every $t \in[\tau, T]$, the function $u_{i}(t, \cdot)$ belongs to the space $S^{\prime}\left(R^{n}\right)$ of tempered distributions on $R^{n}$, and the mapping $t \rightarrow u_{i}(t)$ is continuous in the topology $\sigma\left(S^{\prime}, S\right)$. Indeed, we needed condition 3 only in the proof of equality (67). It is easy to see that $(67)$ can also be obtained using the $S^{\prime}$-condition formulated in this remark.

Theorem 9. Let $\mu \in \mathcal{P}_{n, T}, f \in L^{p}$ with $1<p \leq \infty$, and $0 \leq \tau<T$. Let $u$ be a measurable function on $[\tau, T] \times R^{n}$, satisfying the following conditions:

1. For every $\epsilon$ with $0<\epsilon<T-\tau$, we have $|u(t, x)| \leq M_{\epsilon} \exp \left\{a_{\epsilon}|x|\right\}$, where $(t, x) \in[\tau+\epsilon, T] \times R^{n}$, and $M_{\epsilon}$ and $a_{\epsilon}$ are positive constants depending on $\epsilon$.

2. The function $t \rightarrow u(t, \cdot)$ is a solution to equation $(1)$ in the $D^{\prime}\left((\tau, T) \times R^{n}\right)$ sense.

3. The function $t \rightarrow u(t, \cdot)$ is weakly continuous in $L^{p}$ on the interval $[\tau, T]$ (weak ${ }^{*}$ continuous if $\left.p=\infty\right)$.

4. $u(0, x)=f$ a.e. on $R^{n}$.

Then for every $t$ with $\tau \leq t \leq T$, we have $u(t)=U_{\mu}(t, \tau) f$ a.e. on $R^{n}$.

Remark 16. If $\mu \in \mathcal{P}_{n, T} \cap \mathcal{P}_{n, T}^{*}$, then Theorem 9 holds for $p=1$. A similar theorem also holds for the backward heat equation and the backward propagator $Y_{\mu}$.

Proof of Theorem 9. With no loss of generality we may assume $\tau=0$. Let $\epsilon>0$ and denote $\eta_{\epsilon}(t)=\mu(t+\epsilon)$ and $g_{\epsilon}(t)=u(t+\epsilon)$ for all $0 \leq t \leq T-\epsilon$. Then

$$
\left\{\begin{array}{l}
\frac{\partial g_{\epsilon}}{\partial t}=\frac{1}{2} \Delta g_{\epsilon}-\eta_{\epsilon}(t) g_{\epsilon} \\
g_{\epsilon}(0)=u(\epsilon)
\end{array}\right.
$$

in the $D^{\prime}\left((0, T-\epsilon) \times R^{n}\right)$-sense. Moreover, we have

$$
\sup _{t: 0 \leq t \leq T-\epsilon} \sup _{x \in R^{n}}\left|g_{\epsilon}(t, x)\right| \exp \left\{-a_{\epsilon}|x|\right\}<\infty .
$$

Using Remark 15 and applying Theorem 8 to $g_{\epsilon}(t)$ and $U_{\eta_{\epsilon}}(t, 0) u_{\epsilon}$, we get

$$
\begin{array}{r}
u(\epsilon+t)(x)=U_{\eta_{\epsilon}}(t, 0) u(\epsilon)(x) \\
=U_{\eta_{\epsilon}}(t, 0)(u(\epsilon)-f)(x)+E_{x} f\left(B_{t}\right) \exp \left\{-C_{\mu}(t, t+\epsilon)\right\} \\
=I_{1}+I_{2} .
\end{array}
$$

Now using (43), we see that for every $g \in L^{p^{\prime}}$,

$$
\int_{R^{n}} g(x) U_{\eta_{\epsilon}}(t, 0)(u(\epsilon)-f)(x) d x=\int_{R^{n}}(u(\epsilon)-f) Y_{\eta_{\epsilon}}(t, 0) g d x .
$$

Since $Y_{\eta_{\epsilon}}(t, 0)=Y_{\mu}(t+\epsilon, \epsilon)$, and the family $Y_{\mu}(t, \tau)$ is continuous in $L^{p^{\prime}}$ for $0 \leq$ $\tau \leq t \leq T$ (see Remark 12), the set $\left\{Y_{\eta_{\epsilon}}(t, 0) g\right\}, 0 \leq \epsilon \leq T-t$, is compact in $L^{p^{\prime}}$. It follows from conditions 2 and 3 in Theorem 9 and from (69) that

$$
\lim _{\epsilon \rightarrow 0} I_{1}=0
$$

in the weak topology of $L^{p}$. Now (68), (70) and the conditions in Theorem 9 give

$$
u(t)=\lim _{\epsilon \rightarrow 0+} E_{x} f\left(B_{t}\right) \exp \left\{-C_{\mu}(t, t+\epsilon)\right\}
$$


in the weak topology of $L^{p}$. Hence, the function $u(t)$ can be uniquely reconstructed from the initial condition. Since the function $U_{\mu}(t, 0) f(x)$ satisfies all conditions in Theorem 9 (see theorems 3 and 4 ), we get $u(t)=U_{\mu}(t, 0)$ a.e.

This completes the proof of Theorem 9 .

Theorem 9 also holds for the space $B U C$ instead of the space $L^{p}$. Here we assume that $f \in B U C, u(t) \in B U C$ for $\tau \leq t \leq T$, and that the function $t \rightarrow u(t)$ in condition 3 is weak* continuous in $L^{\infty}$. Similar uniqueness theorem holds for the space $C_{\infty}$.

\section{REFERENCES}

[AS] M. Aizenman and B. Simon, Brownian motion and Harnack's inequality for Schrödinger operators, Comm. Pure Appl. Math. 35 (1982), 209-271. MR84a:35062

[AM] S. Albeverio and Zhi-Ming Ma, Perturbation of Dirichlet forms-lower semiboundedness, closability and form cores, J. Functional Analysis 99 (1991), 332-356. MR.92i:47039

[A] D. G. Aronson, Non-negative solutions of linear parabolic equations, Ann. Scuola Norm. Sup. Pisa 22 (1968), 607-694. MR.55:8553

[BM1] P. Blanchard and Zhi-Ming Ma, Semigroups of Schrödinger operators with potentials given by Radon measures, In: Stochastic Processes-Physics and Geometry (eds. S. Albeverio, et al.), World Scientific, Singapore, 1989.

[BM2] P. Blanchard and Zhi-Ming Ma, New results on the Schrödinger semigroups with potentials given by signed smooth measures, In: Proc. Silvri Workshop (eds. Korezlioglu, et al.), Lecture Notes in Math. 1444, Springer Verlag, Berlin and New York, 1990. MR.91m:35067

[DvC] M. Demuth and J. A. van Casteren, Stochastic Spectral Theory for Selfadjoint Feller Operators. A functional integration approach, Birkhäuser Verlag, Basel, 2000. MR 2002d:47066

[FLP] D. Feyel and A. de La Pradelle, Étude de l'équation $1 / 2 \Delta-u \mu=0$ où $\mu$ est une mesure positive, Ann. Inst. Fourier, Grenoble 38 (1988), 199-218. MR90j:35074

[F] M. Fukushima, Dirichlet Forms and Markov Processes, North-Holland, Amsterdam, 1980. MR:81f:60105

[Ge] R. K. Getoor, Measure perturbations of Markovian semigroups, Potential Analysis 11 (1999), 101-133. MR2001c:60119

[Gu1] A. Gulisashvili, Sharp estimates in smoothing theorems for Schrödinger semigroups, J. Functional Analysis 170 (2000), 161-187. MR2001i:47069

[Gu2] A. Gulisashvili, Classes of time-dependent measures and the behavior of Feynman-Kac propagators, C. R. Acad. Sci. Paris, Ser.I 334 (2002), 1-5. MR2003g:60133

[Gu3] A. Gulisashvili, On the heat equation with a time-dependent singular potential, J. Funct. Anal. 194 (2002), no. 1, 17-52. MR2003i:35117

[GK] A. Gulisashvili and M. A. Kon, Exact smoothing properties of Schrödinger semigroups, Amer. J. Math. 118 (1996), 1215-1248. MR.97m:47053

[H] W. Hansen, Harnack inequalities for Schrödinger operators, Ann. Scuola Norm. Sup. Pisa XVIII (1999), 413-470. MR2001b:31008

[JL] G. W. Johnson and M. Lapidus, The Feynman Integral and Feynman's Operational Calculus, Clarendon Press, Oxford, 2000. MR 2001i:58015

[LP] A. de La Pradelle, Sur les perturbations d'espaces harmonique, Bull. Classe Sci. 6-9 (1990), 201-212. MR.93d:31010

[LS] V. Liskevich and Y. Semenov, Estimates for fundamental solutions of second-order parabolic equations, J. London Math. Soc. 62 (2000), 521-543. MR2002j:35132

[N] M. Nagasawa, Stochastic Processes in Quantum Physics, Birkhäuser, Basel, 2000. MR 2001g:60148

[QZ1] Qi Zhang, On a parabolic equation with a singular lower order term, Transactions Amer. Math. Soc. 348 (1996), 2811-2844. MR.96k:35073

[QZ2] Qi Zhang, On a parabolic equation with a singular lower order term, Part 2: The Gaussian bounds, Indiana Univ. Math. J. 46 (1997), 989-1020. MR98m:35079

[RRSV] F. Räbiger, A. Rhandi, R. Schnaubelt, and J. Voigt, Non-autonomous Miyadera perturbation, Differential Integral Equations 13 (2000), 341-368. MR2002b:34095 
[SV] R. Schnaubelt and J. Voigt, The non-autonomous Kato class, Arch. Math. 72 (1999), 454-460. MR,2000h:35058

[S] B. Simon, Schrödinger semigroups, Bull. Amer. Math. Soc. 7 (1982), 445-526. MR:86b:81001a

[T] A. N. Tikhonov, Théorèmes d'unicité pour l'equation de la chaleur, Mat. Sbornik 42 (1935), 199-216.

[Ti] E. C. Titchmarsh, Introduction to the Theory of Fourier Integrals, Oxford University Press, London, 1937.

[V1] J. Voigt, Absorption semigroups, their generators, and Schrödinger semigroups, J. Functional Analysis 67 (1986), 167-205. MR 88a:81036

[V2] J. Voigt, Absorption semigroups, Feller property, and Kato class, In: Operator Theory: Advances and Applications, 78, Birkhäuser, 1995, 389-396. MR96j:47039

[Z] T. S. Zhang, Generalized Feynman-Kac semigroups, associated quadratic forms and asymptotic properties, Potential Analysis 14 (2001), 387-408. MR2002d:31012

Department of Mathematics, Ohio University, Athens, Ohio 45701

E-mail address: guli@bing.math.ohiou.edu 